\title{
Self-adjuvanting nanoemulsion targeting dendritic cell receptor Clec9A enables antigen-specific immunotherapy
}

\author{
Bijun Zeng, ${ }^{1}$ Anton P.J. Middelberg, ${ }^{2}$ Adrian Gemiarto, ${ }^{3}$ Kelli MacDonald, ${ }^{4}$ Alan G. Baxter, ${ }^{3}$ Meghna Talekar, ${ }^{1}$ Davide Moi, ${ }^{1}$ \\ Kirsteen M. Tullett, ${ }^{5,6}$ Irina Caminschi, ${ }^{5,6,7}$ Mireille H. Lahoud, ${ }^{5,6}$ Roberta Mazzieri, ${ }^{1}$ Riccardo Dolcetti, ${ }^{1,8}$ and Ranjeny Thomas ${ }^{1}$ \\ 'Diamantina Institute, Translational Research Institute, and ²Australia Institute for Bioengineering and Nanotechnology, University of Queensland, Brisbane, Queensland, Australia. ${ }^{3}$ James Cook University, \\ Townsville, Queensland, Australia. ${ }^{4}$ QIMR Berghofer, Brisbane, Queensland, Australia. ${ }^{5}$ Infection and Immunity Program, Monash Biomedicine Discovery Institute and Department of Biochemistry and Molecular \\ Biology, Monash University, Melbourne, Victoria, Australia. ${ }^{6}$ Burnet Institute, Melbourne, Victoria, Australia. ${ }^{7}$ Department of Microbiology and Immunology, The University of Melbourne at the Peter Doherty \\ Institute for Infection and Immunity, Melbourne, Victoria, Australia. ${ }^{8}$ Centro di Riferimento Oncologico -Istituto di Ricovero e Cura a Carattere Scientifico, National Cancer Institute, Aviano, Italy.
}

\begin{abstract}
Non-antigen-specific stimulatory cancer immunotherapies are commonly complicated by off-target effects. Antigen-specific immunotherapy, combining viral tumor antigen or personalized neoepitopes with immune targeting, offers a solution. However, the lack of flexible systems targeting tumor antigens to cross-presenting dendritic cells (DCs) limits clinical development. Although antigen-anti-Clec9A mAb conjugates target cross-presenting DCs, adjuvant must be codelivered for cytotoxic T lymphocyte (CTL) induction. We functionalized tailored nanoemulsions encapsulating tumor antigens to target Clec9A (Clec9A-TNE). Clec9A-TNE encapsulating OVA antigen targeted and activated cross-presenting DCs without additional adjuvant, promoting antigen-specific $\mathrm{CD4}^{+}$and $\mathrm{CD8}{ }^{+} \mathrm{T}$ cell proliferation and $\mathrm{CTL}$ and antibody responses. OVA-Clec9A-TNEinduced DC activation required CD4 and CD8 epitopes, CD40, and IFN- $\alpha$. Clec9A-TNE encapsulating HPV E6/E7 significantly suppressed HPV-associated tumor growth, while E6/E7-CpC did not. Clec9A-TNE loaded with pooled B16-F10 melanoma neoepitopes induced epitope-specific $\mathrm{CD4}^{+}$and $\mathrm{CD8}^{+} \mathrm{T}$ cell responses, permitting selection of immunogenic neoepitopes. Clec9A-TNE encapsulating 6 neoepitopes significantly suppressed B16-F10 melanoma growth in a CD4+ T cell-dependent manner. Thus, cross-presenting DCs targeted with antigen-Clec9A-TNE stimulate therapeutically effective tumor-specific immunity, dependent on T cell help.
\end{abstract}

\section{Introduction}

The clinical success of monoclonal antibody therapy targeting immune checkpoint modulators has stimulated much enthusiasm for anticancer immunotherapy. Although promising, checkpoint inhibitors provide clinical benefit to a limited number of patients, and response is heterogeneous, mainly because of different levels of immunogenicity among cancer subtypes. Moreover, treatment with these drugs is also often associated with the induction of adverse autoimmune reactions due to the lack of specificity of therapeutic effects. There is thus a clinical need to tailor antitumor immune responses more precisely toward the specific and often unique antigens of individual tumors. This possibility is now made practically feasible by advanced genomic sequencing, which identifies the mutational landscape of each individual tumor and specific neoantigens targeted by the host immune system, when released from regulatory checkpoints.

Cancer vaccines are designed to generate tumor-specific T lymphocytes and therefore may complement the capacity of checkpoint inhibitors to reinvigorate tumor-specific T cells (1-5). However, cancer vaccination is challenging (1). Recently, small trials of 2 candi-

Authorship note: RD and RT are co-senior authors.

Conflict of interest: The authors have declared that no conflict of interest exists.

Submitted: August 9, 2017; Accepted: February 20, 2018

Reference information: J Clin Invest. 2018;128(5):1971-1984.

https://doi.org/10.1172/JCI96791. date vaccine approaches to enhance immunity toward tumor neoepitopes - long polypeptides with adjuvant and stimulatory tumor RNA lipoplexes - demonstrated safety, feasibility, and immunogenicity of neoepitope vaccination $(6,7)$. Furthermore, vaccination with E7 peptides and adjuvant was immunogenic and reduced progression of human papillomavirus-driven (HPV-driven) vulval carcinoma (8-10). These approaches deliver vaccine antigens with adjuvant to dendritic cells (DCs), inducing antigen-specific cytotoxic T lymphocytes (CTLs), type I IFN, and antitumor immunity (7). However, such strategies rely on passive targeting of DCs of all subtypes in lymphoid tissues, some of which induce predominantly regulatory responses. Furthermore, because neoepitopes predicted to bind MHC molecules may stimulate regulatory $\mathrm{T}$ cell responses, strategies are needed to enrich for the most immunogenic sequences to induce both $\mathrm{CD} 4^{+}$and $\mathrm{CD} 8^{+}$tumor-specific responses.

Cross-presenting DCs are $\mathrm{BATf} 3^{+} \mathrm{CD} 103^{+} \mathrm{CD} 8^{+}$in mice and CD141 ${ }^{+}$in humans $(11,12)$. They infiltrate tumors and take up antigens released by dying tumor cells and, when signaled by type I IFN, are able to induce strong and specific $\mathrm{CD} 4^{+}$and $\mathrm{CD} 8^{+} \mathrm{T}$ cell responses $(13,14)$. In the absence of BATf3, checkpoint inhibitors and vaccine strategies suppress tumors poorly $(15,16)$. CTL induction requires the activation of $\mathrm{CD} 8$ epitope-loaded DCs with either adjuvant or CD40 signaling by cognate $\mathrm{CD} 40 \mathrm{~L}^{+} \mathrm{CD} 4^{+}$helper $\mathrm{T}$ cells $(17,18)$. Flexible and immunogenic systems targeting tumor antigens to cross-presenting DCs in vivo thus represent the key to induction of $\mathrm{CD}^{+}$and cognate $\mathrm{CD} 4^{+}$tumor-specific responses in 
A

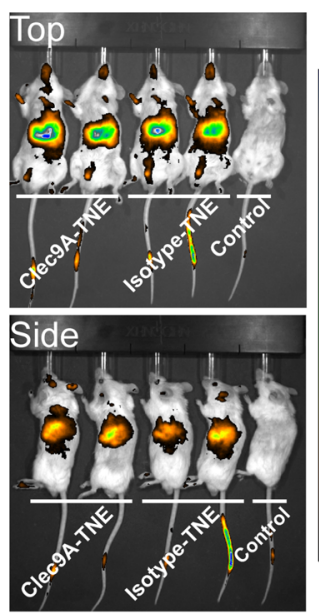

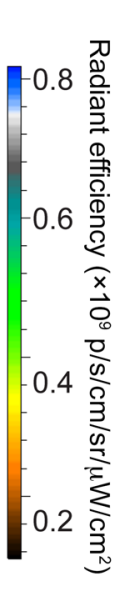

B

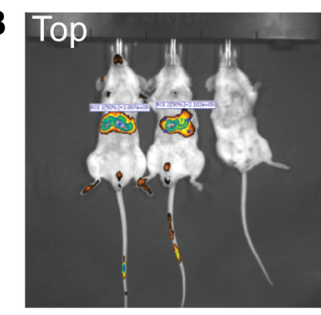

C

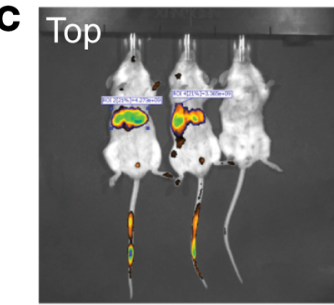

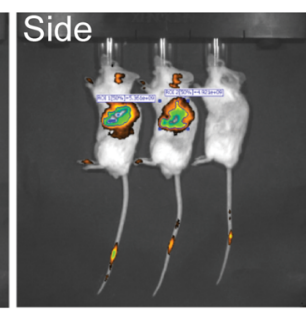

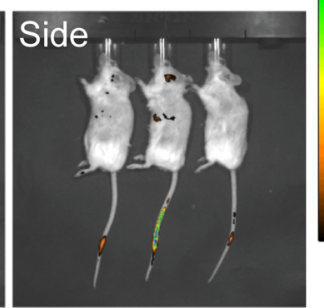

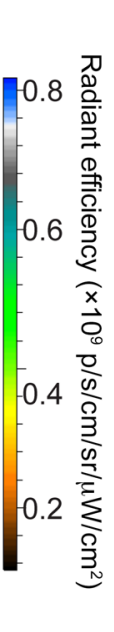

D

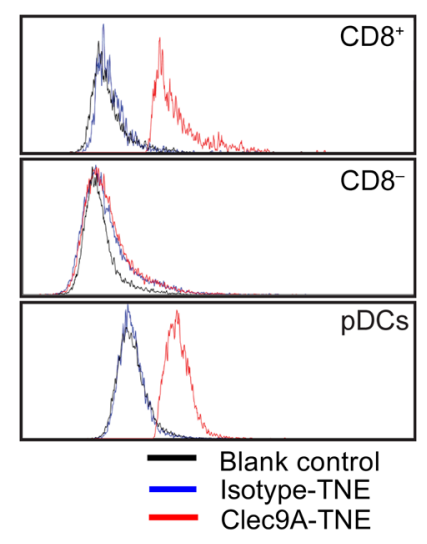

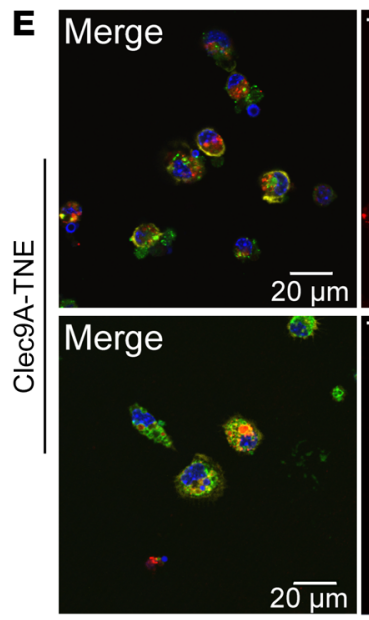
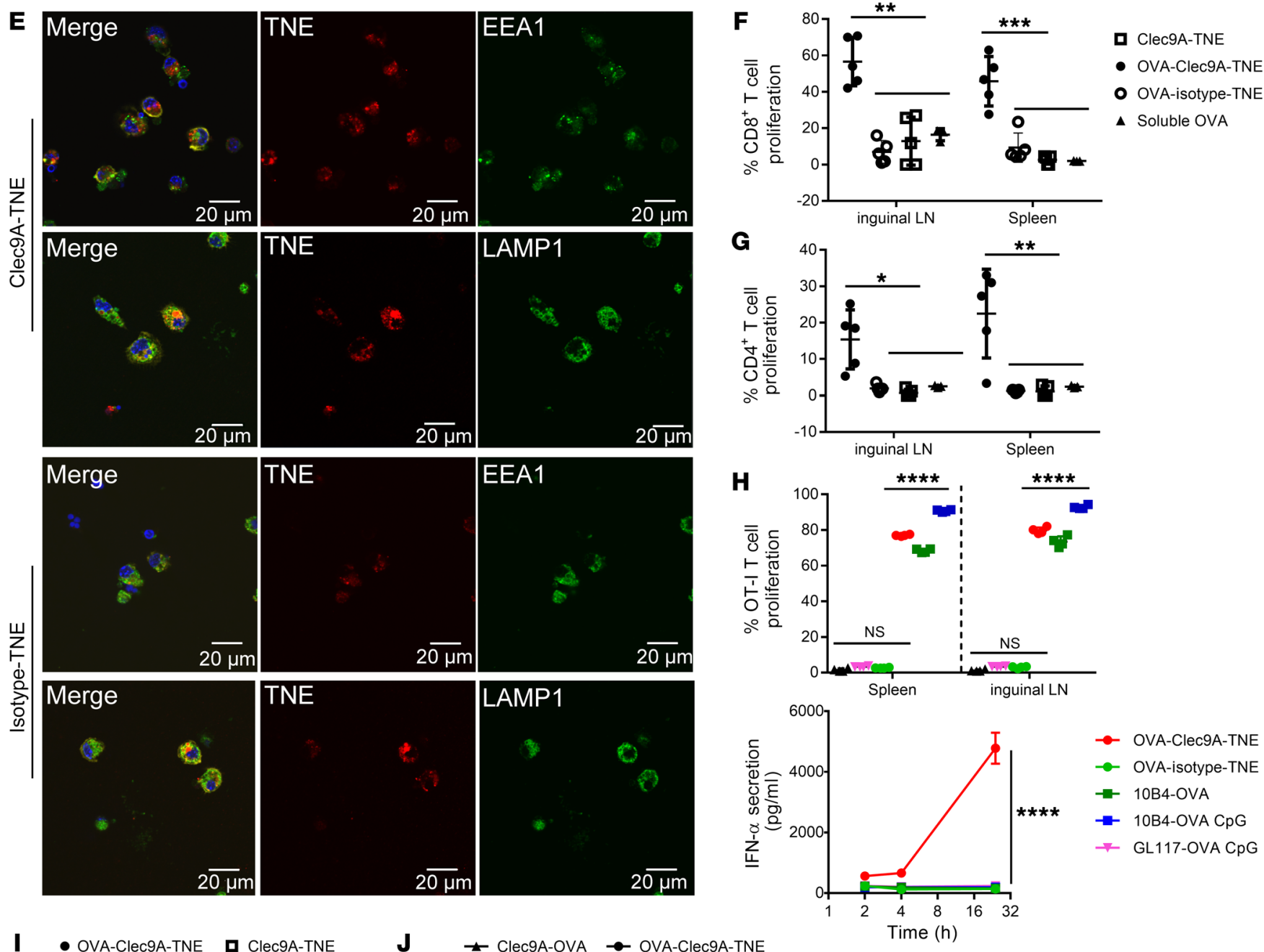

I OVA-Clec9A-TNE a Clec9A-TNE

- OVA-isotype-TNE $\quad$ Sol. OVA

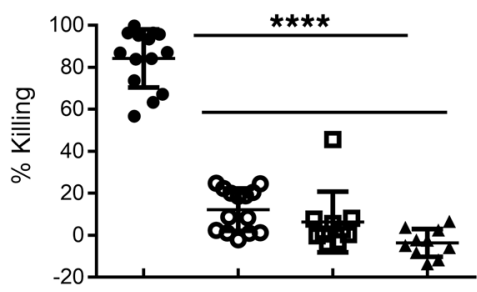

J

$\leftarrow$ Clec9A-OVA $\rightarrow$ OVA-Clec9A-TNE

$\triangle$ isotype-OVA OVA-isotype-TNE

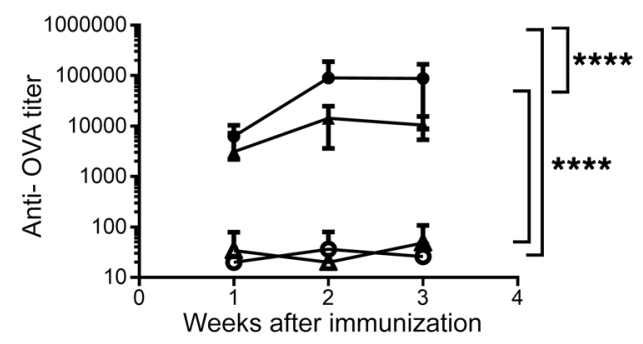


Figure 1. OVA-Clec9A-TNE targets cross-presenting DCs and promotes antigen-specific $T$ cell responses. (A) Images of C57BL/ 6 mice 1 day after i.v. injection of DiR-labeled Clec9A-TNE, isotype-TNE, or free DiR solution as indicated. (B and $\mathbf{C}$ ) Images of $\mathrm{C} 57 \mathrm{BL} / 6$ mice 7 days after i.v. injection of DiR-labeled Clec9A-TNE (B) or DiR-labeled isotype-TNE (C). p/s/cm/sr/ $\mu \mathrm{W} / \mathrm{cm}^{2}$, radiant efficiency, total photons per second per square centimeter per steradian in the irradiance range (microwatts per square centimeter); p, photons; sr, steradian. (D) Binding/uptake of TNE by CD8 ${ }^{+}$CDCs,

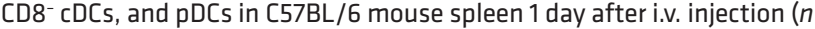
= 3). (E) CD11C $C^{+}$CS sorted from naive C57BL/6 mice were incubated with Dil-labeled Clec9A-TNE or isotype-TNE (red) for 3 hours. Cells were then washed and stained with anti-EEA1 or anti-LAMP1 (green) and DAPI (nuclei, blue). (F and $\mathbf{G}$ ) Proliferation of CD8 ${ }^{+}$OT-I (F) and CD4+ OT-II (C) cells in inguinal lymph nodes (LN) and spleen 6 days after i.v. injection of $5 \mu \mathrm{g}$ of soluble OVA, or $200 \mu$ l of Clec9A-TNE, OVA-Clec9A-TNE, or OVA-isotype-TNE (formulated with $5 \mu \mathrm{g}$ of OVA). (H) OT-I T cell proliferation in spleens of mice 5 days after i.v. injection of OVA-Clec9A-TNE, OVA-isotype-TNE (each formulated with $200 \mathrm{ng}$ of OVA), or $1 \mu \mathrm{g}$ of OVA anti-clec9A^(10B4)-OVA or isotype ${ }^{\wedge}$ (GL117)-OVA fusion protein conjugates without or with CpG $(n=4$ from 2 individual experiments). Serum IFN- $\alpha$ level at 2, 4, and 24 hours after vaccination was measured by ELISA. (I) Percent in vivo OVA-specific killing 5 days after i.v. injection with OVA-Clec9A-TNE or OVA-isotype-TNE (both formulated with $5 \mu \mathrm{g}$ of OVA), Clec9A-TNE, or $5 \mu \mathrm{g}$ soluble OVA ( $n=10-14$ from 3 individual experiments). (J) C57BL/ 6 mice were injected i.v. with $5 \mu \mathrm{g}$ of Clec9A-OVA, OVA-Clec9A-TNE, isotype-OVA, or OVA-isotype-TNE without additional adjuvant. Serum anti-OVA Ig titer was quantified 1,2 , and 3 weeks later by ELISA ( $n=10$ from 2 separate experiments). ${ }^{*} P<0.05$; ${ }^{* *} P<$ $0.01 ;{ }^{* *} P<0.001 ;{ }^{* * *} P<0.0001$ by Tukey's multiple-comparisons test.

the clinic. Current approaches to target cross-presenting DCs have chemically or molecularly conjugated antigen to mAbs recognizing specific endocytic receptors (19-22) such as the C-type lectin receptor Clec9A, or DNGR, which is expressed by cross-presenting DCs and plasmacytoid DCs (pDCs) in mice, and CD141 $1^{+}\left(\mathrm{BDCA3}^{+}\right)$ DCs in humans $(19,23,24)$. In vivo, Clec9A organizes the processing and cross-presentation of dead cell and viral antigens by MHC class I, resulting in CTL induction for viral and tumor immunity $(19,24)$. However, Clec9A-antigen conjugates are challenging to manufacture and require additional costimulation or adjuvant to elicit antitumor immune responses. In fact, although Clec9A conjugates reproducibly induce high titers of specific antibody after a single i.v. inoculation without additional adjuvant, CTL induction requires systemic coadministration of agonist anti-CD40 and/or TLR-agonist adjuvant $(22,24)$, which may increase the risk of systemic inflammatory adverse effects. An alternative approach used a peptide to target Clec9A $\mathrm{A}^{+}$DCs with $\mathrm{OVA}_{257-264}$, but in vivo CTL priming also required adjuvant (25).

Here we combined the concepts of systemic nanoparticle delivery and targeting of antigen to cross-presenting DCs in an alternative approach, in which we encapsulated antigen excipient in a Clec9A-targeting tailorable nanoemulsion (Clec9A-TNE). Oil-inwater nanoemulsion systems have an excellent safety profile and are widely used as clinical vaccine adjuvants (26-30). We reported previously that intraperitoneally injected Clec $9 \mathrm{~A}-\mathrm{TNE}$, grafted with immune-evading polymer PEG and anti-Clec9A mAb using a noncovalent click self-assembly, evaded nonspecific phagocytosis and clearance and targeted antigen to $\mathrm{CD} 8^{+} \mathrm{DCs}$ in mice. OVA encapsulated within the TNE oil core induced antigen-specific $\mathrm{CD}^{+} \mathrm{T}$ cell responses in vitro (31). Although this platform nanocarrier technology showed that targeting of cross-presenting DCs could be achieved, the immunogenicity of TNE-encapsulated antigen was not explored. Here we show unexpectedly, given the lack of DC activation by anti-Clec9A-antigen conjugates, that in the absence of adjuvant, cross-presenting DCs targeted with antigenClec9A-TNE stimulate DC activation, antigen-specific CTLs, and highly effective tumor-specific immunity, dependent on the presence of CD4 helper epitopes and CD40 signaling.

\section{Results}

To target antigen to Clec $9 \mathrm{~A}^{+}$DCs in vivo, we first encapsulated OVA antigen into Clec9A-TNE (OVA-Clec9A-TNE) using a double emulsion method. OVA-Clec9A-TNE had a similar size distribution, as measured by dynamic light scattering, when diluted in water or isotonic PBS (Supplemental Figure 1A; supplemental material available online with this article; https://doi.org/10.1172/ JCI96791DS1), and maintained stability when stored at $4^{\circ} \mathrm{C}$ for up to 256 days (Supplemental Figure 1B). As stability of nanocarriers in physiological conditions is required for effective cellular targeting, the data suggest that OVA-Clec9A-TNE are stable in physiological environments and suitable for i.v. injection. To assess in vivo biodistribution, we labeled Clec9A-TNE and the nontargeting isotype-TNE with the fluorescent lipophilic dye DiR, and injected each preparation i.v. via tail vein into BALB/c mice. Live mice were imaged 24 hours (Figure 1A) and 7 days (Figure 1, B and C) after injection using in vivo imaging. Clec9A-TNE and isotype-TNE but not free dye control accumulated in liver and spleen 24 hours after injection (Figure 1A). At 7 days, Clec9A-TNE accumulated in spleen (Figure 1B), whereas isotype-TNE accumulated in the liver (Figure 1C). Clec9A-TNE but not isotype-TNE were taken up by splenic $\mathrm{CD}^{+}$DCs and pDCs. Both TNEs were taken up to a similar, small extent by CD8- DCs (Figure 1D). Rapid antigen degradation in lysosomal compartments reduces cross-presentation but supports MHC class II-mediated presentation, while antigens delivered to early endosomes are cross-presented efficiently (32, 33). To determine the intracellular localization of Clec9A-TNE, we sorted splenic CD11 $\mathrm{c}^{+}$DCs from naive mice, incubated them with fluorescently labeled Clec9A-TNE or isotype-TNE for 3 hours, then stained early endosomes and lysosomes using the cell compartment markers EEA1 and LAMP1, respectively. Internalized Clec9A-TNE colocalized with EEA1 and LAMP1, while isotype-TNE colocalized with LAMP1 only (Figure 1E). These data indicate that, after recognition by the Clec9A receptor, TNEs are internalized and reach early endosomes and lysosomes. In contrast, isotype-TNE are not efficiently taken up by the cells (Figure $1 \mathrm{E})$, but the low levels that are internalized reach only lysosomes.

OVA and other antigens conjugated to anti-Clec9A mAb to target $\mathrm{CD} 8^{+}$DCs have been shown to induce antigen-specific $\mathrm{CD} 4^{+}$ and $\mathrm{CD} 8^{+} \mathrm{T}$ cell proliferation and strong antibody responses in mice, but not DC activation (19). We tested the capacity of OVA-Clec9ATNE to induce proliferation of antigen-specific $\mathrm{T}$ cells and their CTL activity in the absence of adjuvant. CellTrace Violet-labeled (CTV-labeled) $\mathrm{CD}^{+}$(OT-I) or $\mathrm{CD}^{+}$(OT-II) OVA-specific T cell receptor transgenic $\mathrm{T}$ cells were adoptively transferred to B6.SJL-Pt$\mathrm{prc}^{\mathrm{a}}$ mice, followed by i.v. injection of OVA-Clec9A-TNE, OVA-isotype-TNE, empty Clec9A-TNE, or the same quantity of soluble OVA as delivered in TNE. Six days after immunization with OVAClec9A-TNE, the proliferative response of transferred antigen-spe- 


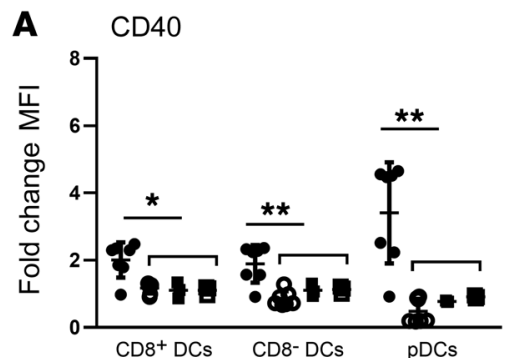

B

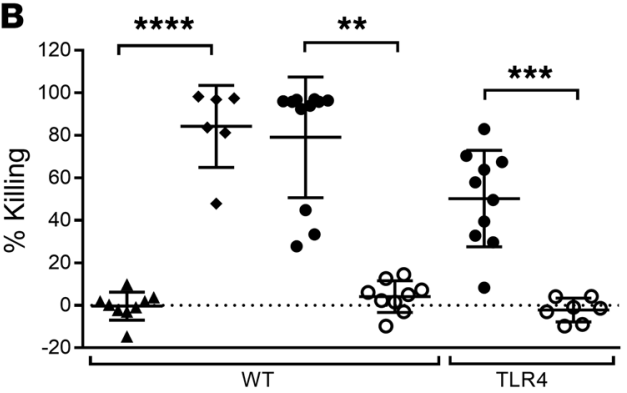

D

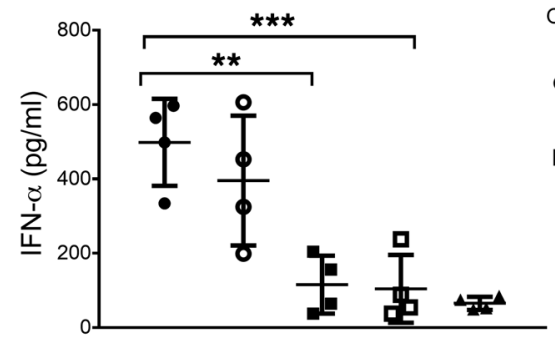

- WT

- CASP1-

- IFNAR ${ }^{-1}$

D MyD88 $8^{-1}$
CD80

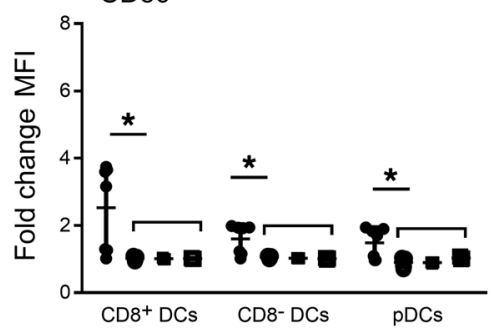

- OVA-Clec9A-TNE

- OVA-isotype-TNE

- Clec9A-TNE

口 Isotype-TNE

Untreated

- Clec9A-ova poly I:C

- OVA-Clec9A-TNE

- OVA-isotype-TNE

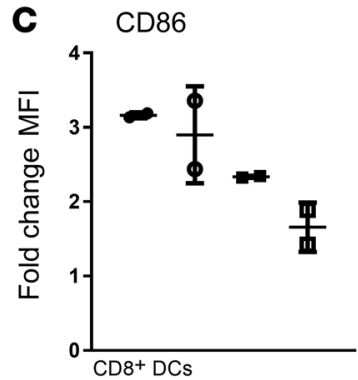

$\mathbf{E}$

OVA-Clec9A-TNE in:

A WT, untreated

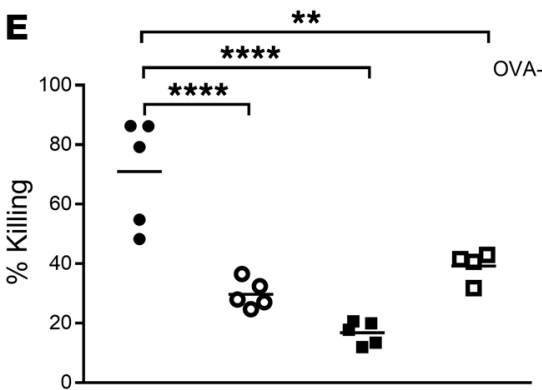

Figure 2. OVA delivered by Clec9A-TNE promotes MyD88-dependent DC activation and IFN- $\alpha$ production. (A) C57BL/6 mice were injected i.v. with OVAClec9A-TNE, OVA-isotype-TNE, Clec9A-TNE, or isotype-TNE. Six hours later, surface expression of CD86, CD80, and CD40 by CD8+ DCs, CD8- DCs, and pDCs was analyzed by flow cytometry ( $n=6$ from 2 separate experiments). (B) C57BL/6 or TLR4 ${ }^{-1-}$ mice were adoptively transferred with equal numbers of unpulsed CFSE ${ }^{10}$ and SIINFEKL-pulsed CFSE ${ }^{\text {hi }}$ target cells 6 days after i.v. injection with OVA-Clec9A-TNE or OVA-isotype-TNE. The percentage of SIINFEKL peptide-specific lysis in spleen is depicted ( $n=7-12$ from 3 separate experiments). (C and D) C57BL/6, Casp1 $1^{-/-}$, IFNAR1 $1^{-1-}$, MyD88 $8^{-/-}$, or CD40 injected i.v. with OVA-Clec9A-TNE. Six hours later the expression of CD86 by CD8 ${ }^{+}$splenic DCs was analyzed by flow cytometry (C) ( $n=7$ from 2 experiments), and IFN- $\alpha$ levels in serum from these mice were measured by ELISA (D). (E) C57BL/6 (WT), CASP1 $1^{-1-}$, IFNAR1 $1^{-1-}$, MyD88 $8^{-1-}$, or CD40 $0^{-/-}$mice were adoptively transferred with equal numbers of unpulsed CTV ${ }^{10}$ and SIINFEKL-pulsed CTVhi target cells 5 days after i.v. injection with OVA-Clec9A-TNE ( $n=9-10$ from 2 experiments). Specific killing of target cells is shown. ${ }^{*} P<0.05 ;{ }^{* *} P<0.01 ;{ }^{* *} P<0.001 ;{ }^{* * *} P<0.0001$ by Tukey's multiple-comparisons test.

cific $\mathrm{CD} 4^{+}$and $\mathrm{CD} 8^{+} \mathrm{T}$ cells, as determined by dilution in $\mathrm{CTV}$ fluorescence intensity, was significantly increased relative to mice immunized with OVA-isotype-TNE or empty Clec9A-TNE or mice injected with the same amount of soluble OVA (Figure 1, F and G). We compared OVA-Clec9A-TNE in the same assay as anti-Clec9AOVA and isotype-OVA fusion protein conjugates without or with $\mathrm{CpG}$ adjuvant. Only preparations targeting OVA to Clec9A induced OVA-specific $\mathrm{CD}^{+} \mathrm{T}$ cell proliferation. The $\mathrm{CD} 8^{+} \mathrm{T}$ cell response was significantly greater in response to OVA-Clec9A-TNE than to Clec9A-OVA conjugate. Clec9A-OVA plus CpG stimulated significantly higher proliferation. Despite this, only OVA-Clec9A-TNE stimulated production of systemic IFN- $\alpha$, detectable in serum within 24 hours (Figure 1H). OVA $257-264$ (SIINFEKL) is the dominant OVA CTL epitope in C57BL/6 mice and is commonly used to investigate CTL-mediated killing of target cells. Five days after immunization with OVA-Clec9A-TNE, OVA-isotype-TNE, empty Clec9A-TNE, or soluble OVA, recipients were injected with an equal mix of syngene- ic SIINFEKL-pulsed splenic target cells labeled with $5 \mu \mathrm{M}$ of CTV $\left(\mathrm{CTV}^{\mathrm{hi}}\right)$ and unpulsed syngeneic splenocytes labeled with $0.5 \mu \mathrm{M}$ of CTV $\left(\mathrm{CTV}^{\mathrm{lo}}\right)$. Twenty hours later, residual SIINFEKL-specific target cells were enumerated relative to unpulsed splenocytes in recipient mice. After immunization with OVA-Clec9A-TNE, approximately $80 \%$ of SIINFEKL-specific targets were killed (Figure 1I). In contrast, no OVA-specific killing was induced in mice immunized with nontargeting OVA-isotype-TNE or soluble OVA. Given that Clec9A-TNE traffic to both endosomes and lysosomes and induce $\mathrm{CD} 4^{+} \mathrm{T}$ cell proliferation in vivo, we compared anti-OVA antibody induction by OVA-Clec9A-TNE, OVA-isotype-TNE, and OVA conjugated to anti-Clec9A or isotype mAb (10B4-OVA, GL117-OVA). Each group received an equivalent i.v. dose of $5 \mu \mathrm{g}$ of OVA. The anti-OVA response induced by Clec9A-OVA conjugate was significantly greater than the response to isotype-OVA, and the response to OVA-Clec9A-TNE was significantly greater than the response to OVA-isotype-TNE and Clec9A-OVA conjugate (Figure 1J). 


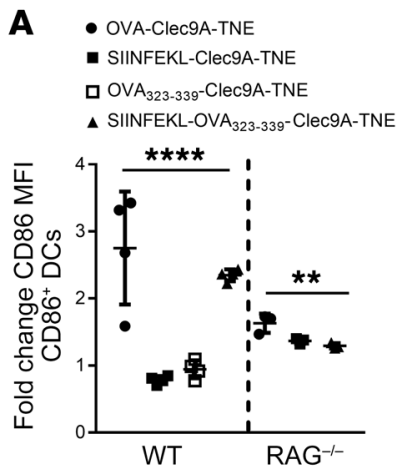

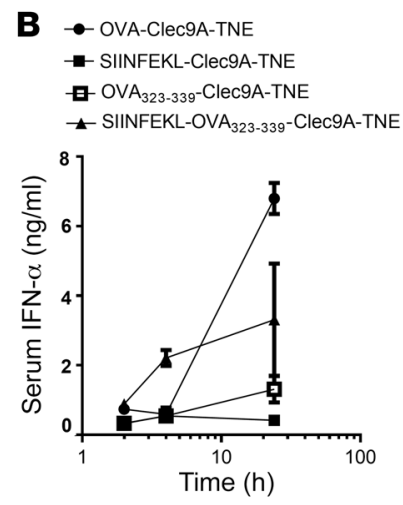

$\mathbf{F}$
C

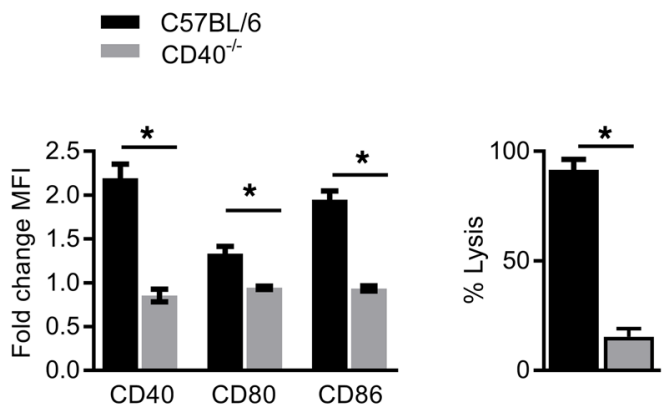

E

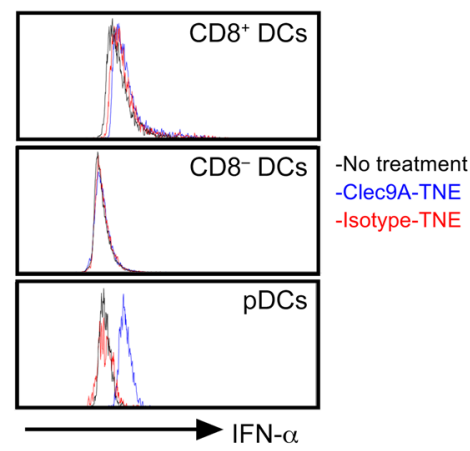

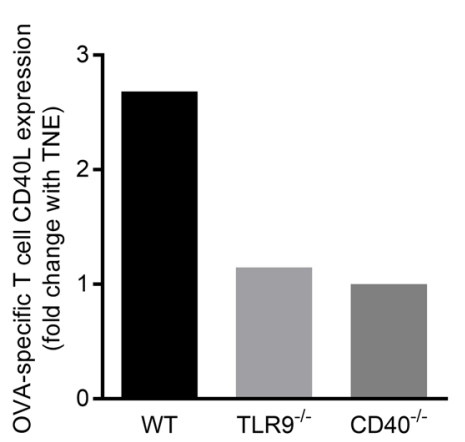

G

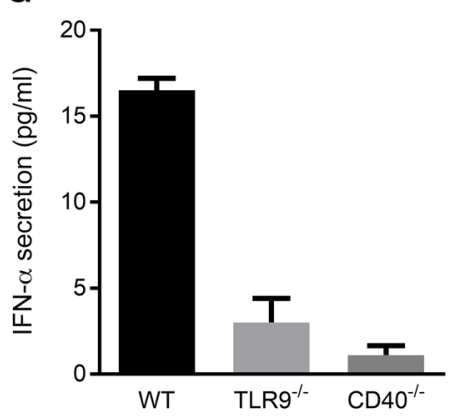

Figure 3. OVA-Clec9A-TNE-mediated DC activation and IFN- $\alpha$ production require CD4 ${ }^{+}$and $C D 8^{+} T$ cell epitope presentation and CD40L-CD40 crosstalk. (A)

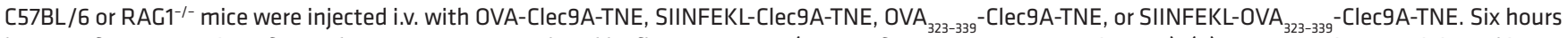
later, surface expression of $\mathrm{CD} 86$ by CD8 ${ }^{+} \mathrm{DCs}$ was analyzed by flow cytometry ( $n=3-8$ from 2 separate experiments). (B) C57BL/6 mice were injected i.v. with OVA-Clec9A-TNE, SIINFEKL-Clec9A-TNE, OVA ${ }_{323-339}$-Clec9A-TNE, SIINFEKL-OVA ${ }_{323-339}$-Clec9A-TNE, or OVA-isotype-TNE. Serum was collected 2, 4, and 24 hours after injection, and IFN- $\alpha$ levels were quantified by ELISA $(n=4)$. (C) C57BL/6 or CD40 $1 /-$ mice were injected i.v. with OVA-Clec9A-TNE. Six hours later, surface expression of CD40, CD80, and CD86 by CD8 $8^{+}$C s was analyzed by flow cytometry $(n=4)$. (D) C57BL/6 or CD40 $0^{-/-}$mice were adoptively transferred with equal numbers of unpulsed CFSE ${ }^{10}$ and SIINFEKL-pulsed CFSE hi target cells 6 days after i.v. injection with OVA-Clec9A-TNE. The percentage of SIINFEKL peptide-specific lysis in spleen is depicted $(n=4)$. (E) Splenic CD11 ${ }^{+}$DCs were purified from C57BL/6 mice, then cultured for 5 hours with OT-I OVA-specific $\mathrm{CD}^{+}$and OT-II OVA-specific CD4+ ${ }^{+}$cells, in the presence or absence of OVA-Clec9A-TNE or OVA-isotype-TNE. Histograms depict intracellular IFN- $\alpha$ levels in gated DC populations. Splenic CD11c $\mathrm{DCs}$ were purified from C57BL/6 (WT), CD40-1-, or TLR9-/- mice, then cultured for 24 hours with OT-I OVA-specific CD8 and OT-II OVA-specific CD4+ T cells, in the presence or absence of OVA-Clec9A-TNE or OVA-isotype-TNE. (F) Increase in CD40L expression by OT-II cells in the presence of OVA-Clec9A-TNE relative to no TNE control. (G) Increase in IFN- $\alpha$ secretion into cell culture supernatant in the presence of OVA-Clec9A-TNE relative to no TNE control. Representative data from 6 mice are shown. ${ }^{*} P<0.05 ;{ }^{* *} P<0.01 ;{ }^{* * *} P<0.0001$ by Tukey's multiple-comparisons test.

Given the immunogenicity of OVA antigen delivered to DCs when loaded into Clec9A-targeting TNE relative to nontargeting isotype-TNE after i.v. injection in the absence of adjuvant, we determined whether DCs were activated after OVA-Clec9A-TNE administration. OVA-Clec9A-TNE, OVA-isotype-TNE, empty Clec9A-TNE, or isotype-TNE were administered to mice, and 6 hours later splenic DC maturation markers were analyzed by FACS. After injection of OVA-Clec9A-TNE, we observed a surprising increase in CD86, CD80, and CD40 expression by CD8 ${ }^{+} \mathrm{DCs}$, CD8- DCs, and pDCs. OVA-isotype-TNE, empty Clec9A-TNE, and isotype-TNE controls did not affect expression of these DC maturation markers (Figure 2A and Supplemental Figure 2). Given almost exclusive binding of OVA-Clec9A-TNE to $\mathrm{CD} 8^{+} \mathrm{DCs}$ and $\mathrm{pDCs}$, the upregulation of maturation markers by Clec $9 \mathrm{~A}^{-} \mathrm{CD} 8^{-} \mathrm{DC}$ suggested that ligation of Clec9A and delivery of OVA may have promoted a systemic cytokine response and secondary DC activation. Furthermore, the strong adjuvant effect of OVA-Clec9A-TNE compared with empty Clec9A-TNE or OVA-isotype-TNE indicated that OVA antigen could only activate DCs when delivered in a Clec9A-targeted system. In contrast, Clec9A-OVA conjugate was shown pre- viously not to activate DCs after i.v. injection $(19,22)$, suggesting that the TNE delivery system is particularly immunogenic but only when antigen is encapsulated. Furthermore, coadministration of adjuvant - anti-CD40 alone or with poly I:C - with Clec9A-OVA conjugate was required for induction of CTL responses $(22,24)$. To determine the mechanism of DC activation and CTL induction by OVA-Clec9A-TNE, we first hypothesized that recombinant OVA, which contained sufficient endotoxin to stimulate an NF- $\kappa$ B-GFP reporter cell line (Supplemental Figure 3), activated the TLR4 pathway through ligation of endosomal TLR after Clec9A-mediated nanoparticle uptake and delivery of OVA to the endosome (34), thereby promoting DC activation and CTL induction. To test this, we constructed OVA-Clec9A-TNE using endotoxin-free OVA, and compared CTL induction by OVA-Clec9A-TNE in TLR $4^{-/-}$and WT littermate control mice. OVA-specific CTLs were induced to a similar extent by OVA-Clec9A-TNE without additional adjuvant and Clec9A-OVA conjugate codelivered with poly I:C in littermate controls (Figure 2B). Thus OVA-Clec9A-TNE induced specific CTLs even when OVA was endotoxin-free. Moreover, OVA-specific CTLs were not significantly changed when OVA-Clec9A-TNE 
A
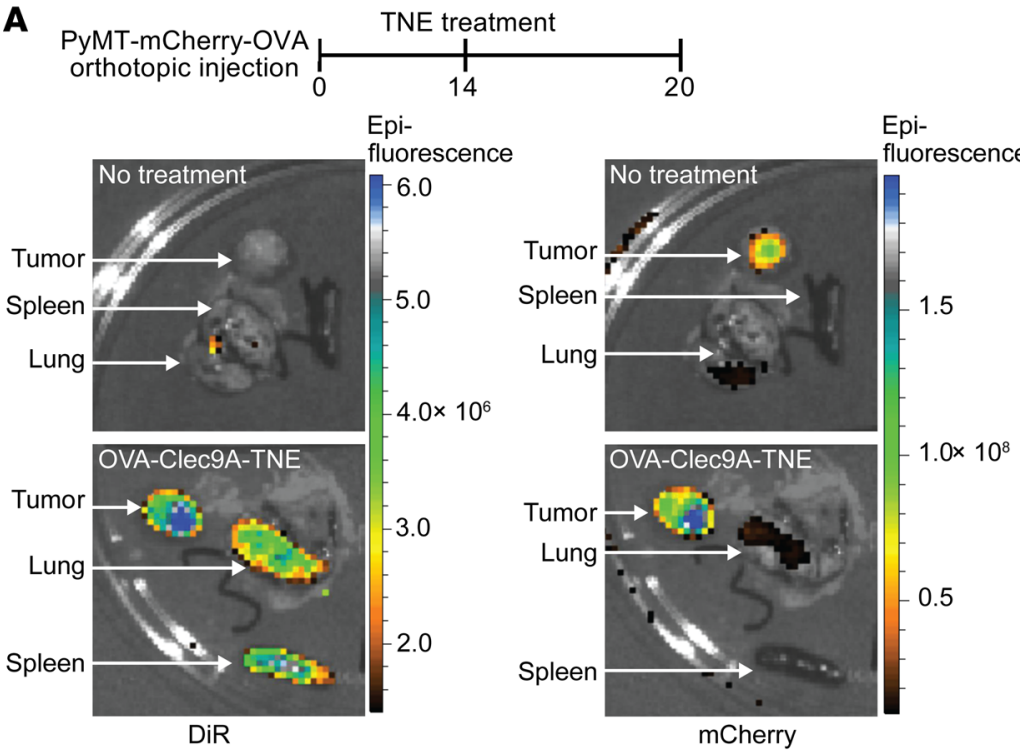

B

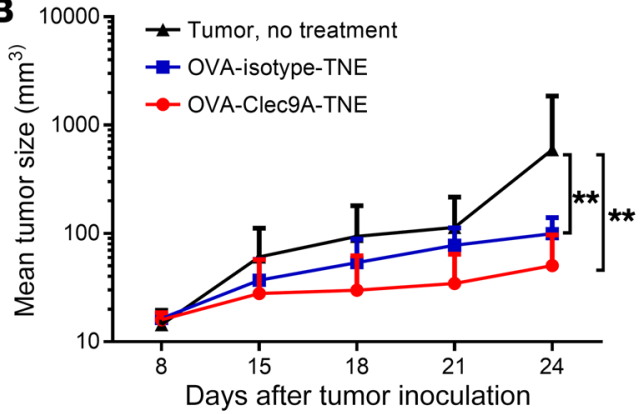

C
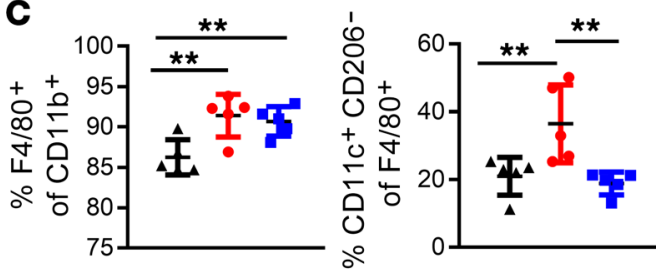

D
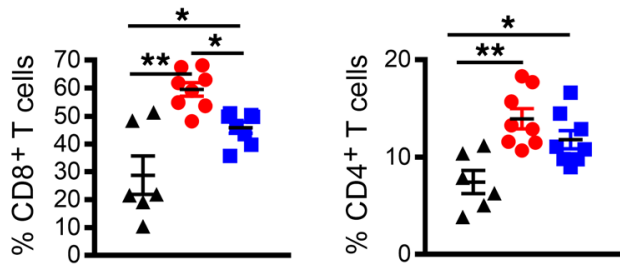

$\leftarrow$ No treatment, tumor only

$\rightarrow$ OVA-isotype-TNE

$\rightarrow$ OVA-Clec9A-TNE

$\rightarrow$ 10B4-OVA

$\rightarrow$ 10B4-OVA Cpg

$\because$ GL117-OVA

- GL117-OVA CpG
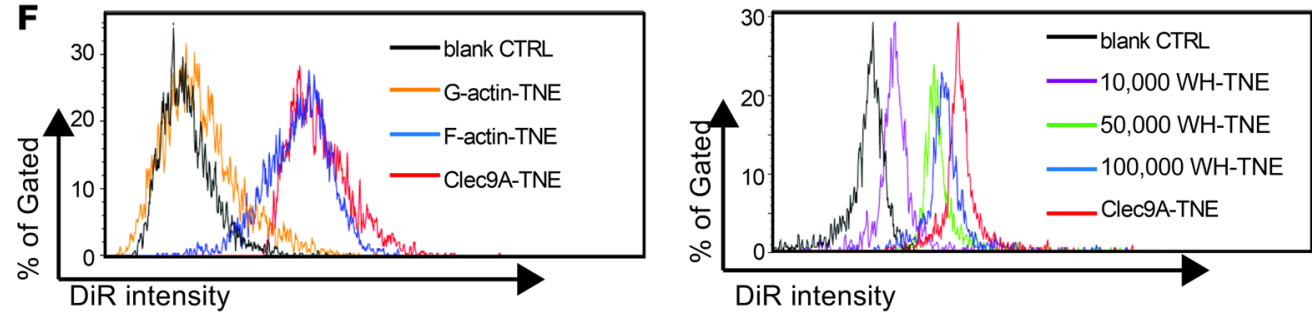

Figure 4. OVA-Clec9A-TNE target lymphoid organs and PyMT-mChOVA tumor and promote a proinflammatory tumor environment associated with tumor control. (A) Organs were harvested from untreated or OVA-Clec9A-TNE-treated PyMT-mChOVA tumor-bearing mice. Images show distribution of DiRlabeled OVA-Clec9A-TNE in lung, tumor, and spleen, and colocalization with mCherry-expressing tumor. (B) C57BL/6 mice $(n=6-8)$ developing tumor after orthotopic injection of PyMT-mCherry-OVA were treated with OVA-Clec9A-TNE or OVA-isotype-TNE or left untreated. Mean tumor size is plotted over time for each group. (C and D) Percentages of M1-like CD11 ${ }^{+}$CD206- macrophages, total F4/80+CD11b macrophages (C), and CD8 ${ }^{+}$and CD4 ${ }^{+}$T cells (D) in tumor were analyzed by FACS, 6 days after TNE injection. (E) C57BL/ 6 mice $(n=7)$ inoculated with $10^{6}$ AT3-OVA tumor cells and 10 days later injected i.V. once with OVA-TNE or OVA-mAb fusion conjugates with or without $10 \mu \mathrm{g} C \mathrm{PC}$ ODN $1668 \mathrm{CpC}$ adjuvant, as indicated. Survival curves of tumor-bearing mice are shown. (F) C57BL/6 mice were injected i.v. with DiR-labeled G-actin-TNE, F-actin-TNE, or Clec9A-TNE (left panel) or 10,000 WH-TNE, 50,000 WH-TNE, 100,000 WH-TNE, and Clec9A-TNE (right panel); 16 hours later, splenocytes were fixed, and then DiR fluorescence intensity was analyzed, indicating uptake of DiR ${ }^{+}$ TNE by CD8 ${ }^{+}$CDCs. Representative of 4 mice per group. ${ }^{*} P<0.05$; ${ }^{* *} P<0.01$ by 2 -way ANOVA test. Survival analyses used the Mantel-Cox log-rank test.

were delivered to TLR4 ${ }^{-/}$mice (Figure 2B), indicating that OVAClec9A-TNE promote induction of CTLs in a TLR4-independent manner. To determine whether MyD88/TRIF-mediated, inflammasome-mediated, or type I IFN-mediated signaling pathways were involved in OVA-Clec9A-TNE-induced DC activation, we screened expression of $\mathrm{CD} 86$ by $\mathrm{CD} 8^{+} \mathrm{CDCs} 6$ hours after in vivo delivery of OVA-Clec9A-TNE. CD86 induction was impaired in IFNAR $^{-/}$and MyD88/Trif ${ }^{-/}$but not caspase- $1^{-/-}\left(\mathrm{Casp1}^{-/-}\right)$mice relative to WT mice (Figure 2C). Consistent with the IFN dependence of this DC activation, serum IFN- $\alpha$ increased 8-fold relative to untreated WT mice within 6 hours after injection of OVA-Clec9A-TNE (Figure 2D). This rapid burst of IFN- $\alpha$ suggests secretion by activated pDCs, as observed in Figure 2A (35). OVA-Clec9A-TNE did not induce IFN- $\alpha$ when delivered to IFNAR $^{-/}$and MyD88/Trif $/-$mice, consistent with positive-feedback activation of $\mathrm{CD} 8^{+}$DCs and pDCs by type I IFN, or crosstalk 
A
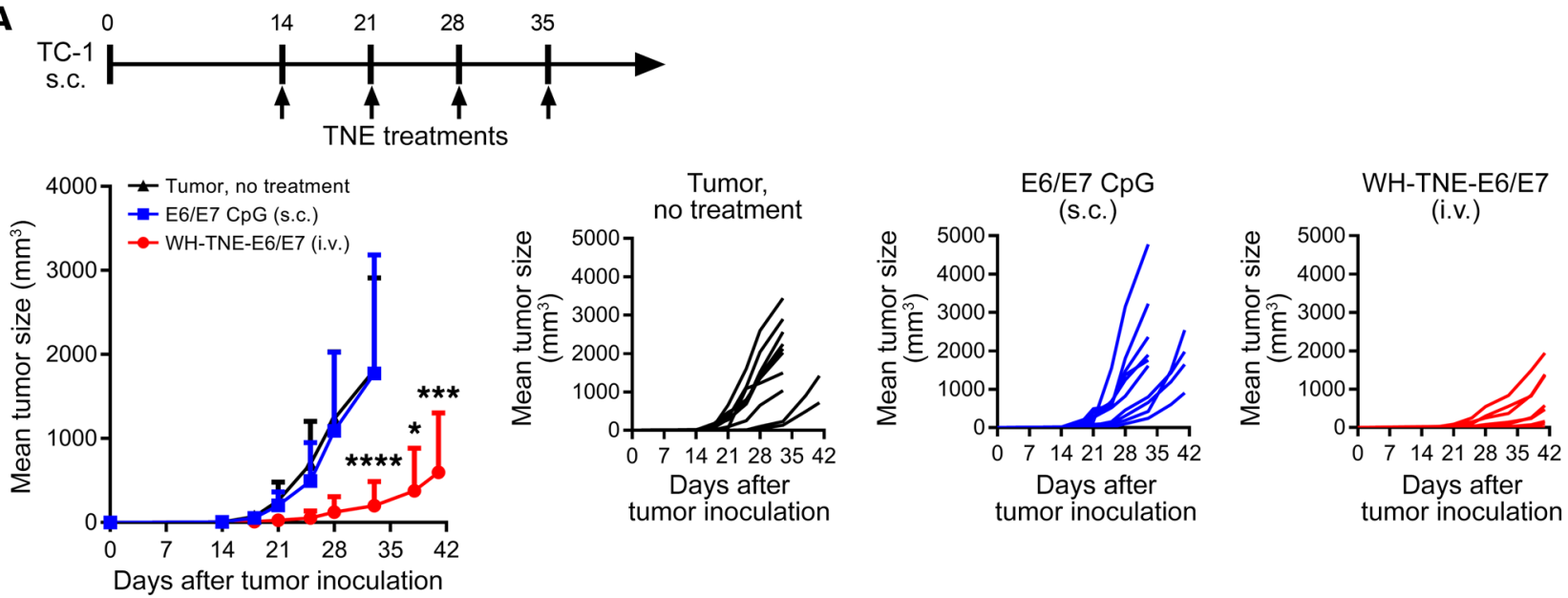

B

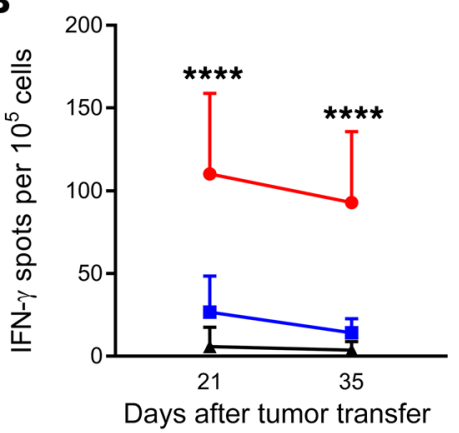

C

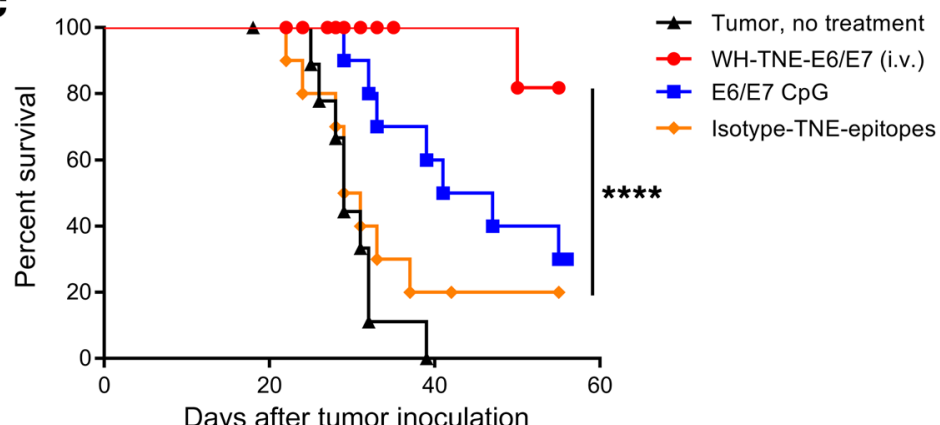

D

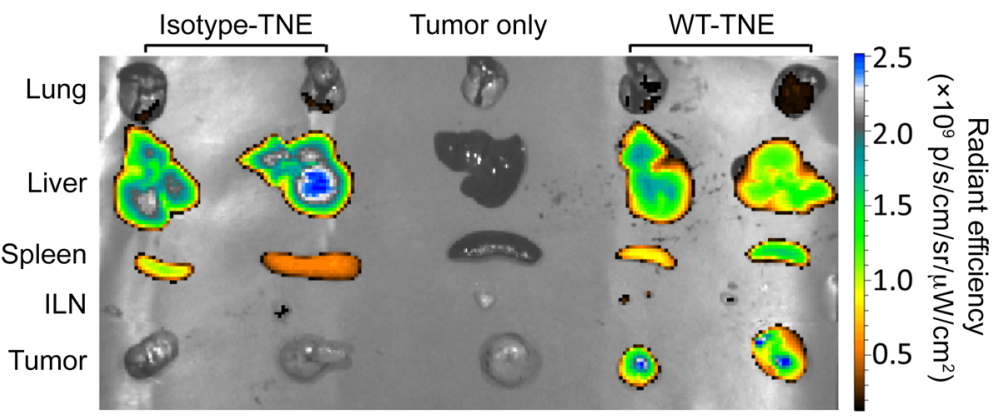

Figure 5. E6/E7-Clec9A-TNE but not E6/E7-CpG vaccination is immunogenic and controls TC1 tumor growth. (A) C57BL/6 mice ( $n=10)$ developing tumor after s.c. injection of TC1 were treated with E6/E7-WH-TNE or E6/E7 with $10 \mu \mathrm{g}$ CpG or left untreated. Mean tumor size is plotted over time for each group, and individual mice are shown. (B) Peripheral blood mononuclear cells were stimulated with HPV16 E7 aa49-57 (RAHYNIVTF) peptide, and IFN- $\gamma$ production was quantified by ELISPOT. (C) Experimental design as in A; survival curves of tumor-bearing mice are shown. (D) Organs were harvested from untreated, DiR-labeled E6/ E7-WH-TNE-treated, or DiR-labeled E6/E7-isotype-TNE-treated TC1 tumor-bearing mice. Images show distribution of DiR-labeled TNE in lung, liver, spleen, inguinal lymph node (ILN), and tumor. ${ }^{*} P<0.05 ;{ }^{* * *} P<0.001 ;{ }^{* * *} P<0.0001$ by 2 -way ANOVA test. Survival analyses used the Mantel-Cox log-rank test.

between DCs (Figure 2D). These data indicate that delivery of OVA promotes MyD88/TRIF-dependent and type I IFN-mediated DC activation, when targeted to Clec $9 \mathrm{~A}^{+} \mathrm{DCs}$ in the absence of adjuvant. Consistent with a requirement of DC activation for the induction of CTLs, low levels of lytic activity were observed after delivery of OVA-Clec9A-TNE to recipient mice lacking the capacity for inflammasome-mediated, type I IFN-mediated, or MyD88/ TRIF-mediated activation (Figure 2E).

CTL induction requires either the activation of DCs presenting a CD8 epitope with adjuvant or costimulatory CD40-mediated crosstalk from $\mathrm{CD} 4^{+} \mathrm{T}$ cells $(17,36)$. Since we had observed delivery of OVA-Clec9A-TNE to early and late endosomes in vitro and the induction of both CD4 and CD8 responses in vivo, we explored whether concomitant delivery of CD4 and CD8 epitopes in Clec9A-TNE would be sufficient to activate DCs in vivo. We encapsulated synthetic sterile SIINFEKL OVA $_{257-264}$ CD8 epitope, OVA $_{323-339}$ CD4 epitope, or both epitopes in Clec9A-TNE. Both OVA-Clec9A-TNE and Clec9ATNE encapsulating the CD4 and CD8 epitopes, but neither OVA $_{323-339}$-Clec9A-TNE nor SIINFEKL-Clec9A-TNE, activated DCs (Figure 3A). Furthermore, there was no difference in DC activation by OVA-Clec9A-TNE, Clec9A-TNE encapsulating 
A

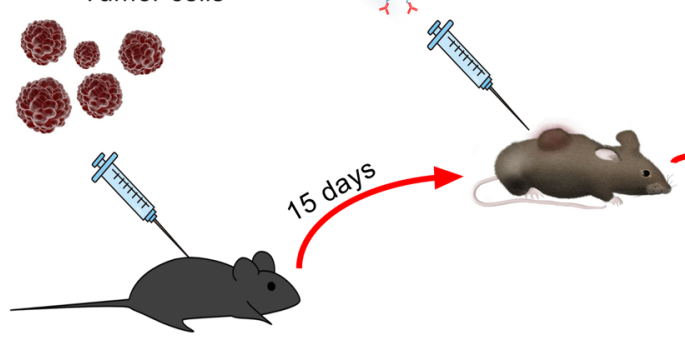

$\uparrow$

B

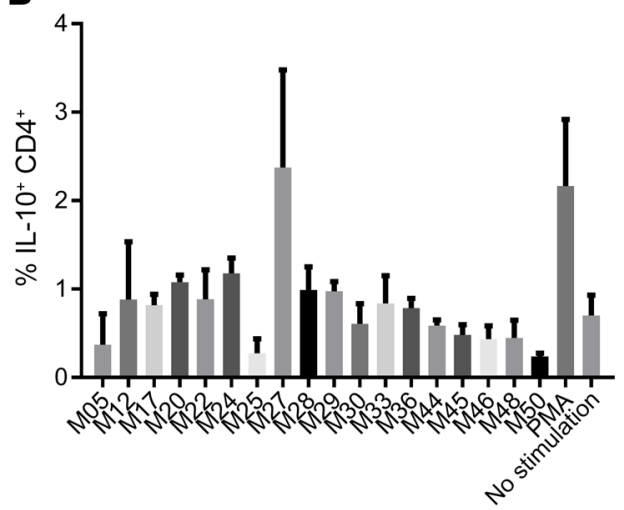

D

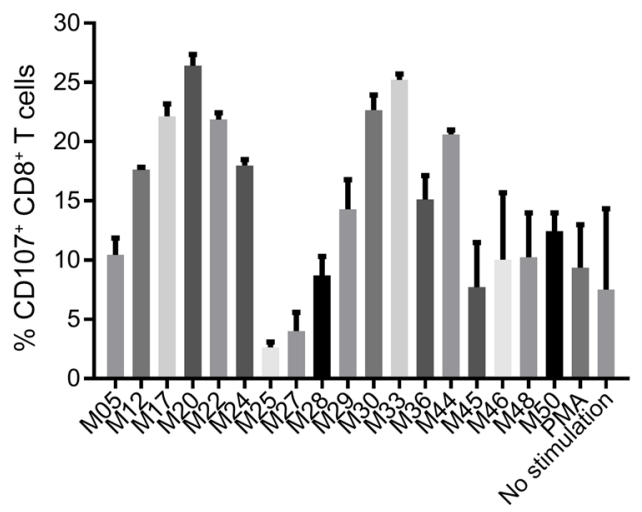

Clec9A-TNE-multiAgs

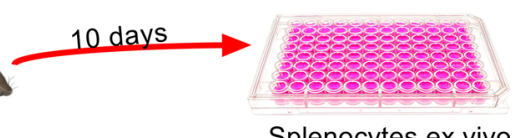

Splenocytes ex vivo restimulation with individual epitope

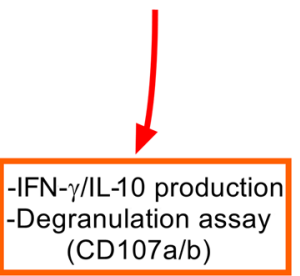

Figure 6. Clec9A-TNE encapsulating neoepitopes generate neoepitopespecific $\mathrm{CD}^{+}{ }^{+}$and $\mathrm{CD8}{ }^{+} \mathrm{T}$ cell responses, which can be used to select immunogenic epitopes. (A) Schematic describing selection of B16-F10 immunogenic epitopes by immunogenicity testing. (B-E) C57BL/6 mice $(n=3)$ inoculated s.c. with B16-F10 were treated with Clec9A-TNE loaded with a pool of B16-F10 mutated epitopes. Splenocytes were harvested 15 days after treatment and restimulated with individual epitopes from the pool. Expression of IL-10 (B), IFN- $\gamma$ (C), and CD107a/b ( $\mathbf{D}$ and $\mathbf{E}$ ) in T cells was quantified by FACS.
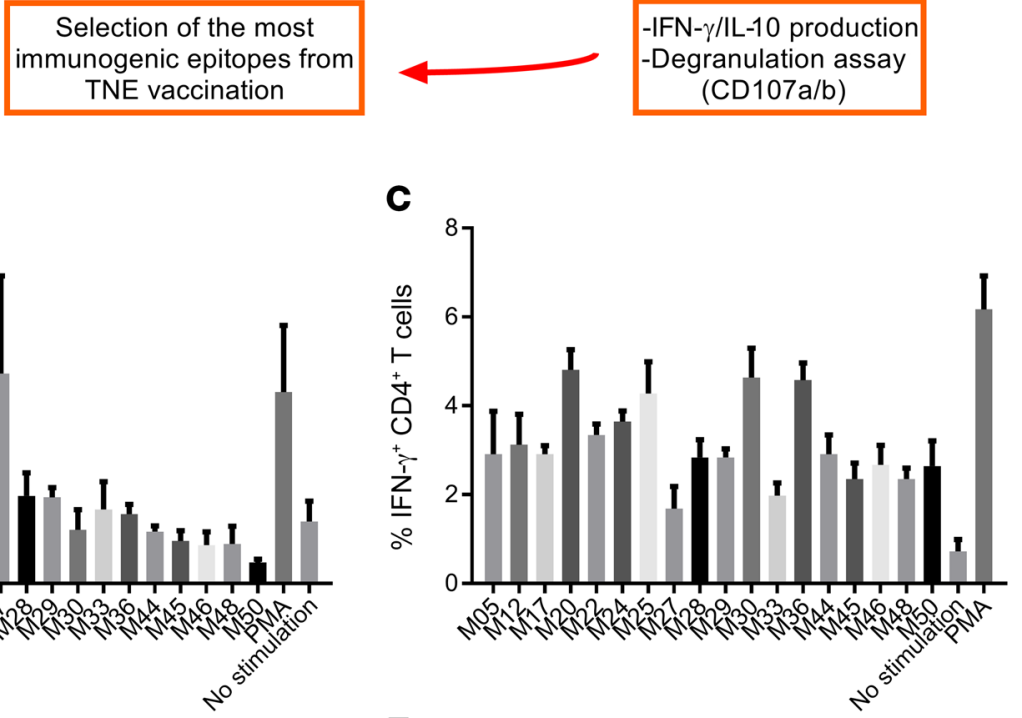

E

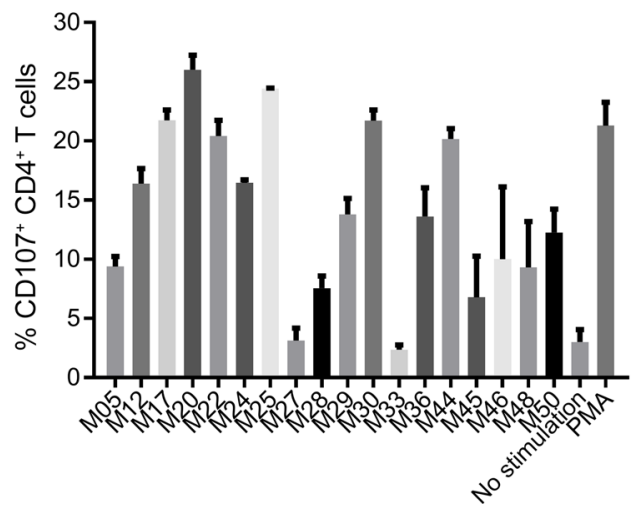

the CD4 and CD8 epitopes, or SIINFEKL-Clec9A-TNE in $\mathrm{RAG1}^{-/-}$mice, which lack $\mathrm{T}$ cells (Figure 3A). Clec9A-TNE encapsulating poly I:C or LPS in the absence of antigen activated DCs much less effectively (Supplemental Figure 4). CD4 helper epitopes enhance DC activation though CD154-CD40 interactions. Furthermore, OVA-Clec9A-TNE and Clec9ATNE encapsulating both CD4 and CD8 epitopes promoted secretion by pDCs of high serum levels of IFN- $\alpha$ (Figure 3B). Consistent with the requirement for CD154-CD40-mediated $\mathrm{T}$ cell help, neither DC activation nor OVA-specific CTLs were induced after delivery of OVA-Clec9A-TNE to CD40 (Figure 3, C and D). When CD11 $\mathrm{c}^{+}$DCs were incubated with OVA-specific $\mathrm{CD}^{+}$and $\mathrm{CD} 8^{+} \mathrm{T}$ cells in the presence or absence of OVA-Clec9A-TNE, IFN- $\alpha$ was expressed only by pDCs (Figure $3 \mathrm{E}$ ). pDCs secrete a burst of IFN- $\alpha$ in response to CpG DNA sequences, for which signaling of TLR9 in early endosomes is required $(37,38)$. To test the requirement of TLR9 and CD4OL/ CD40 signaling for the activation of DCs and IFN- $\alpha$ secretion, we incubated WT, CD40 ${ }^{-/}$, or $\mathrm{TLR}^{-/-} \mathrm{CD} 11 \mathrm{c}^{+} \mathrm{DCs}$ with OVA-specific $\mathrm{CD} 4^{+}$and $\mathrm{CD} 8^{+} \mathrm{T}$ cells in the presence or absence 
A
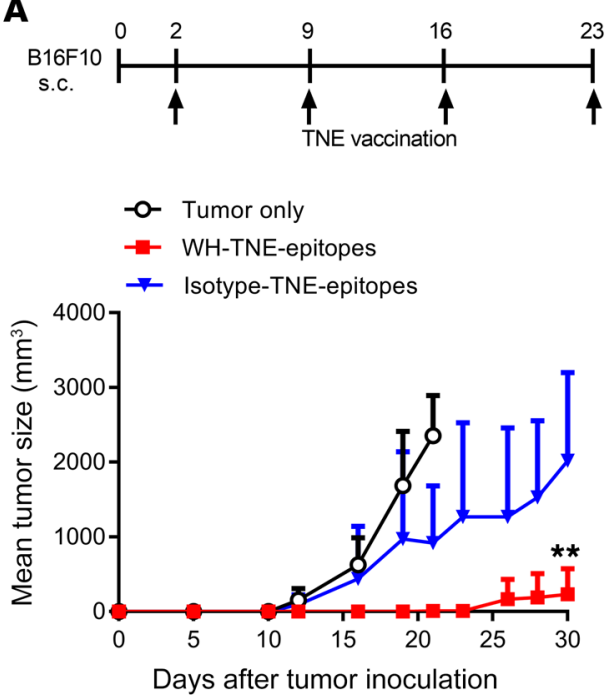

B

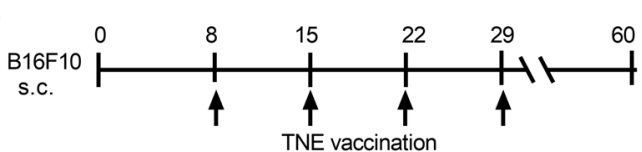

$\rightarrow$ WH-TNE-epitopes

$\rightarrow$ Isotype-TNE-epitopes

-o- Tumor only, no treatment

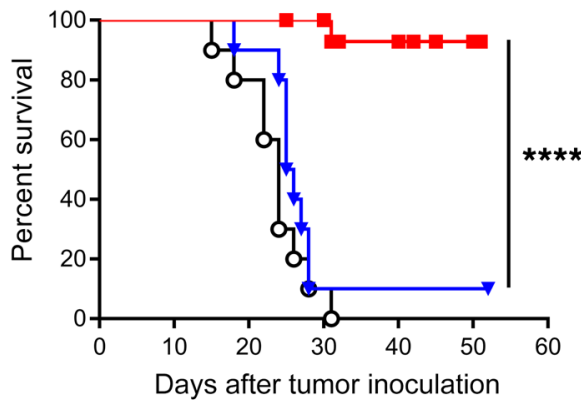

Tumor only,

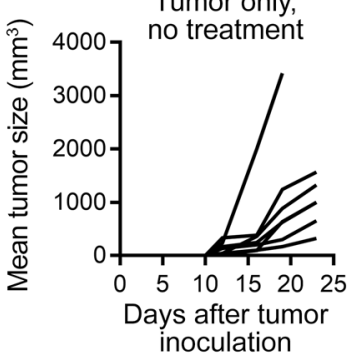

WH-TNE-epitopes

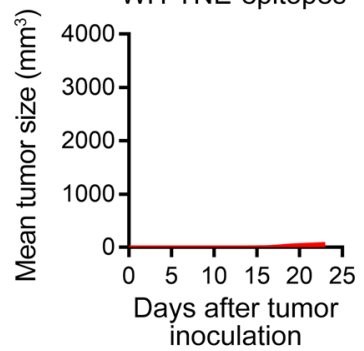

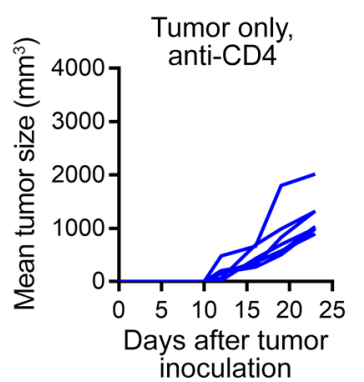

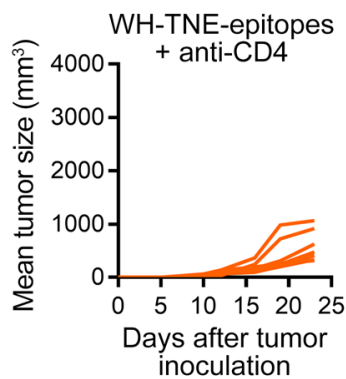

C B16 epitopes

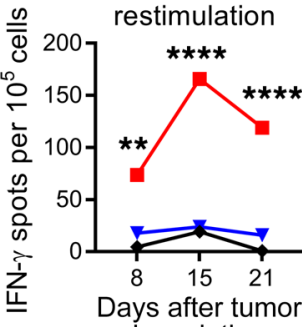
inoculation

Survivin peptide

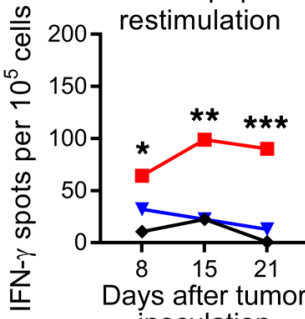
inoculation
D
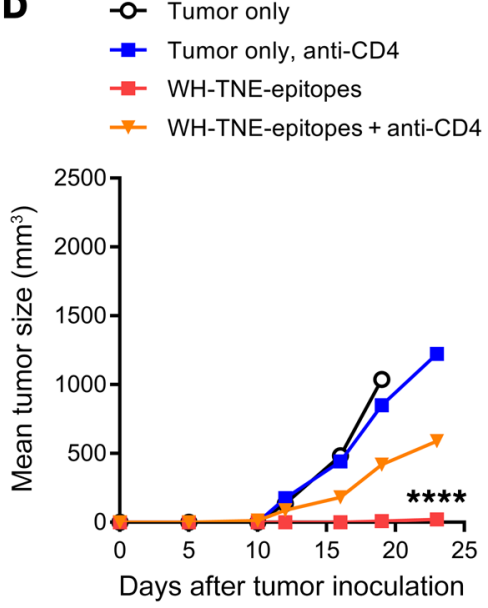

Figure 7. Clec9A-TNE encapsulating immunogenic neoepitopes generate antigen-specific $T$ cell responses and suppress the growth of B16-F10 tumors. (A) C57BL/6 mice $(n=7)$ were inoculated s.c. with B16-F10, then treated with WH-TNE or G-actin-TNE loaded with a pool of B16-F10 mutated epitopes, as described in Methods, or left untreated. Mean tumor size is plotted over time for each group. (B) C57BL/6 mice $(n=10)$ were inoculated s.c. with B16-F10, then treated with WH-TNE or G-actin-TNE loaded with a pool of B16-F10 mutated epitopes or left untreated 8 days after tumor inoculation. Survival curves of tumor-bearing mice are shown. (C) Peripheral blood mononuclear cells were stimulated with B16 epitopes or endogenous survivin peptide, and IFN- $\gamma$ production was measured by ELISPOT. (D) Tumor growth ( \pm CD4-depleting antibody) in mice inoculated s.c. with B16-F10 and treated with WH-TNE loaded with the pool of B16-F10 mutated epitopes or left untreated $(n=7) .{ }^{*} P<0.05 ;{ }^{* *} P<0.01 ;{ }^{* * *} P<0.001 ;{ }^{* * *} P<0.0001$ by 2 -way ANOVA test. Survival analyses used the Mantel-Cox log-rank test.

of OVA-Clec9A-TNE. The OVA-Clec9A-TNE promoted increased CD4OL expression by OVA-specific CD $4^{+} \mathrm{T}$ cells and IFN- $\alpha$ secretion into the supernatant in a CD40- and TLR9-dependent manner (Figure 3, $\mathrm{F}$ and $\mathrm{G}$ ). Taken together, our data indicate that after Clec9A-targeted OVA antigen delivery, $\mathrm{CD} 8^{+} \mathrm{DCs}$ are activated as a result of type I IFN release and antigen-specific CD40-CD4OLmediated $\mathrm{CD} 4^{+} \mathrm{T}$ cell help, to induce antigen-specific CTLs without additional adjuvant. Activation of IFN- $\alpha$ secretion requires CD40 signaling as well as TLR9. The latter is most likely activated as the TNEs reach the early endosome $(37,38)$.
These data indicate that Clec9A-TNE are a self-adjuvanting vaccine platform that simultaneously promotes $\mathrm{CD} 4^{+}$and $\mathrm{CD} 8^{+} \mathrm{T}$ cell responses to delivered antigen, which would be ideal for cancer immunotherapy. To test this, OVA-Clec9A-TNE or OVA-isotypeTNE were administered i.v. once to C57BL/6 mice, 14 days after orthotopic transplant to the mammary fat pad of PyMT-ChOVA breast cancer cells. Six days later, DiR-labeled OVA-Clec9A-TNE were detected in spleen, as expected (Figure 4A). In the primary tumor and lung metastasis, DiR signal colocalized with mCherry, consistent with demonstrated tumor infiltration by $\mathrm{CD} 8^{+} \mathrm{DCs}$ (39). 
Tumor growth was significantly inhibited in mice treated either with OVA-Clec9A-TNE or with OVA-isotype-TNE, but inhibition was greater with OVA-Clec9A-TNE (Figure 4B). The clinical effect of OVA-Clec9A-TNE was associated with significantly greater infiltration by $\mathrm{CD} 11 \mathrm{c}^{+} \mathrm{F} 4 / 80^{+} \mathrm{CD} 206^{-}$inflammatory DCs and $\mathrm{CD} 4^{+}$ and $\mathrm{CD}^{+} \mathrm{T}$ cells (Figure $4, \mathrm{C}$ and D, and Supplemental Figure 5). In the AT3-OVA model of breast cancer, survival of mice was significantly greater after a single administration of OVA-Clec9A-TNE than Clec9A-OVA or isotype-OVA conjugates with or without $\mathrm{CpG}$ adjuvant, or OVA-isotype-TNE (each mouse received $5 \mu \mathrm{g}$ OVA) (Figure 4E). These data indicate that OVA-Clec9A-TNE are taken up within tumors, promote an inflammatory tumor environment, and prolong the survival of tumor-bearing mice.

Since clinical translation may be hampered by immunogenicity of the Clec9A mAb, we exploited the highly conserved F-actin component of the cellular cytoskeleton and a recently described $\mathrm{WH}$ peptide as specific Clec9A ligands to functionalize nanoparticles targeting either mouse or human cross-presenting DCs (25, 40). OVA-WH-TNE had a similar size distribution to OVA-Clec9ATNE, and maintained stability in serum conditions when stored at $4^{\circ} \mathrm{C}$ for up to 25 days (Supplemental Figure $1 \mathrm{C}$ ). In vivo uptake of F-actin-TNE or WH-TNE by mouse CD8 ${ }^{+}$DCs and pDCs was similar to that of Clec9A-TNE, while control G-actin-TNE were not taken up (Figure 4F). Specificity was confirmed by the lack of binding of Clec9A-TNE, F-actin-TNE, or WH-TNE to CD8 DCs (Supplemental Figure 6). Moreover, mice immunized with F-actin-TNE, or WH-TNE loaded with OVA, generated antigen-specific proliferative and cytotoxic responses similar to those of OVA-Clec9A-TNE (Supplemental Figure 7).

To test the efficacy and immunogenicity of Clec9A-TNE for the oncogenic human papillomavirus 16 (HPV16) antigen E6/E7, we generated WH-TNE encapsulating recombinant HPV16 E6/E7 protein and commenced weekly immunization 14 days after s.c. implantation of TC1 tumors. While weekly E6/E7 and CpG adjuvant had no significant impact on tumor growth, WH-E6/E7-TNE significantly suppressed TC1 tumor growth (Figure 5A), induced strong E6/E7-specific IFN- $\gamma$ responses in peripheral blood 21 and 35 days after tumor implantation (Figure 5B), and significantly increased survival of tumor-bearing mice (Figure 5C). After tumors became established, we injected DiR-labeled WH-E6/ E7-TNE i.v. to assess biodistribution. Consistent with evidence that $\mathrm{CD}^{+}$DCs infiltrate tumors (39) and that Clec9A-TNE promote an inflammatory tumor environment (Figure 4, C and D), WH-Clec9A-TNE but not G-actin-TNE localized to tumors as well as spleen and liver, while isotype-TNE localized only to spleen and liver (Figure 5D). These data indicate that Clec9A-TNE effectively target antigen to tumor-infiltrating DCs.

While oncogenic virus expression generates predictable immune target antigens suitable for tumor vaccines, neoepitopes derived from somatic tumor mutations constitute an alternative source of vaccine antigens. In order to exploit peptide neoepitopes for vaccination of the murine B16-F10 melanoma model, we first developed a system to rank immunogenicity of individual neoepitopes using WH-TNE. B16-F10 melanoma cells were implanted s.c., and mice were immunized 15 days later with WH-TNE loaded with a pool of 18 previously described C57BL/6 B16 neoepitopes (41). After 10 days, splenocytes were harvested and restimulated with individual neoepitopes, to compare IL-10 (Figure 6, A and B) and IFN- $\gamma$ (Figure 6C) production by $\mathrm{CD}^{+}{ }^{+} \mathrm{T}$ cells and expression of $\mathrm{CD} 107$ by $\mathrm{CD}^{+}$and $\mathrm{CD} 4^{+} \mathrm{T}$ cells (Figure 6, D and $\mathrm{E}$ ) as a marker of degranulation. Regulatory epitopes were identified as low stimulators of IFN- $\gamma$ and CD107 and high stimulators of IL-10, and were discarded. The resultant stimulatory pool, consisting of 6 neoepitopes (five CD4 neoepitopes, MUT20, MUT25, MUT30, MUT36, and MUT44, and one CD8 neoepitope, MUT33, with highest immunogenicity), was encapsulated into WH-TNE. Two days after s.c. implantation of B16-F10 melanoma, mice were immunized with WH or control G-actin TNE encapsulating the 6-neoepitope pool. WH-neoepitope-TNE but not G-actin-neoepitope-TNE significantly suppressed tumor growth and enhanced survival (Figure 7, A and B) and induced strong IFN- $\gamma$ immunity to pooled B16 epitopes and to the universal tumor antigen, survivin (ref. 42 and Figure 7C). Immunogenicity and antitumor effects of the WH-neoepitope-TNE vaccine were Th cell-dependent, as the significant tumor suppression observed with WH-TNE was lost if $\mathrm{CD}^{+} \mathrm{T}$ cells were depleted during treatment (Figure 7D). These data indicate that immunization with WH-neoepitope-TNE stimulates epitope-specific and additional tumor antigen-specific immunity, as well as tumor suppression, in a CD4 ${ }^{+} \mathrm{T}$ cell-dependent manner. Notably, in the same experiment, all tumor-free mice $(n=5)$ previously treated with WH-neoepitope-TNE were protected by subsequent challenge with B16-F10 cells, consistent with the induction of a memory response.

\section{Discussion}

The Clec9A receptor has been shown to mediate efficient delivery of antigens derived from necrotic cells to cross-presenting DCs. Virally infected cells undergo lysis, thereby exposing the Clec9A ligand F-actin. CD8 ${ }^{+}$DCs take up antigen from dying cells via Clec9A, then shuttle F-actin-bound viral proteins into the cross-presentation pathway (43). We show here that Clec9A-TNE are a highly efficient and immunogenic system for targeted antigen delivery to early endosomes and lysosomes in cross-presenting DCs, which can be exploited for generation of CTLs and cognate $\mathrm{T}$ cell help using protein antigen or pooled CD4 and CD8 tumor neoepitope peptides. Accordingly, CTLs and high titers of antibody were induced after a single systemic administration of OVA-Clec9A-TNE. In contrast, when delivered as a conjugate, anti-Clec9A-antigen efficiently induced specific antibody, but CTLs were not induced without concomitant adjuvant or anti-CD40 delivery, commensurate with the lack of effect of the conjugate on DCs in vivo $(19,24)$. In contrast, we found that a single administration of antigen-Clec9A-TNE activated DCs and induced secretion of IFN- $\alpha$ and specific CTLs very efficiently without additional adjuvant in vivo in a manner dependent on presentation of CD4 and CD8 cognate epitopes, the presence of $\mathrm{CD} 4^{+} \mathrm{T}$ cells, and expression of CD40, MyD88, and IFNAR. Our data are consistent with a model in which, after Clec9A-targeted antigen delivery, CD8 ${ }^{+}$DCs are sufficiently activated as a result of pDC type I IFN release and antigen-specific CD40-CD40L-mediated CD $4^{+} \mathrm{T}$ cell help to induce antigen-specific CTLs without additional adjuvant. Of particular interest, $\mathrm{CD}^{+}$tumor-infiltrating DCs are also targeted by antigen-Clec9A-TNE, leading to local immune acti- 
vation, including greater infiltration by inflammatory DCs than myeloid suppressor cells and $\mathrm{CD} 4^{+}$and $\mathrm{CD} 8^{+} \mathrm{T}$ cell infiltration. Furthermore, in contrast to passive DC-targeting strategies (7, 44-46), a single administration of OVA-Clec9A-TNE inhibited the growth of and enhanced survival with the relatively immunogenic PyMT-OVA and AT3-OVA breast cancer models. After vaccine administration, the burst of IFN- $\alpha$ provoked systemic activation of $\mathrm{CD}^{+}$and $\mathrm{CD} 8^{-}$DCs. IFN- $\alpha$ is a critical predictor of immunogenic presentation of antigen and antitumor responses after anthracycline chemotherapy and RNA lipoplexes $(7,47)$. Indeed, stimulation of IFN- $\alpha$ secretion emerged as the key difference between targeting Clec9A as an OVA conjugate or functionalized on TNE. Crosstalk between DCs is essential in vivo for efficient CTL induction. While pDCs cross-present weakly, if at all, they are known to produce large amounts of IFN- $\alpha$ when signaled through TLR9 and CD40, which in turn activates CD8 ${ }^{+}$ and CD8 ${ }^{-}$DCs for efficient antigen presentation $(48,49)$. Based on previous observations, we speculate that TLR9 activation is triggered as the TNEs reach the early endosome $(37,38)$.

Our findings indicate that the Clec9A-targeting vaccination platform can efficiently exploit the immunogenicity of the unique epitopes expressed by oncogenic viruses or generated by somatic mutations, which represent the most potent antigens for cancer immunotherapy. Indeed, HPV16 synthetic long peptides used within conventional vaccination approaches induced strong antitumor immunity and clinical responses in patients with HPV16-driven carcinomas (50-52). More recently, neoantigen vaccines in the form of RNA polyepitopes or based on the use of synthetic long peptides administered with adjuvant were shown to elicit robust specific $\mathrm{T}$ cell responses to advanced melanoma, with potential clinical benefits $(53,54)$. The ability of the TNE vaccination platform to deliver tumor antigens directly to cross-presenting Clec $9 \mathrm{~A}^{+} \mathrm{DC}$ in vivo and to induce therapeutically relevant $\mathrm{CD}^{+}$and $\mathrm{CD} 4^{+} \mathrm{T}$ cell responses without the addition of adjuvant has the potential to improve the clinical efficacy of personalized cancer immunotherapy. The improved potency of this vaccination strategy is supported by the observation that the majority of mice vaccinated with WH-neoepitope-TNE monotherapy were cured of the highly aggressive B16-F10 melanoma and survived for over 50 days. Rational combinations with immune checkpoint inhibitors may further increase the rate of complete responses and enhance the control of recurrent diseases, as shown in preclinical settings and clinical trials $(2,3,5)$.

Although neoantigen identification has become feasible and affordable by the advances in massively parallel sequencing, algorithms for the prediction of HLA class I and II binding of mutated peptides are only partly reliable (55). Our results emphasize the need to functionally validate the immunogenicity of candidate neoepitopes in order to select the optimal pool of neoantigens to be used for effective vaccination. Elimination of epitopes with regulatory potential seems to be of particular relevance, as shown by the ability of carefully selected B16-F10 neoantigens to induce therapeutically relevant immune responses and to eradicate the tumor in most of the treated animals. The strength of antitumor immune response induced by WH-neoepitope-TNE is also supported by the observation that tumor-free mice were protected by a subsequent challenge with live B16 melanoma cells consistent with induction of a memory response.
Versatile, personalized, antigen-specific cancer vaccines are a long-sought therapeutic strategy in cancer immunotherapy. Clec9A TNE represent such a platform to deliver recombinant tumor protein or neoepitope antigens specifically to cross-presenting DCs. This platform can fully exploit the neoepitope target repertoire for personalized immunotherapeutic approaches.

\section{Methods}

Materials and mice. AM1 (molar mass 2,473, 95\% purity) (56), WH peptide (WPRFHSSVFHTHGGGK), 4T1, and B16-F10 mutated epitopes ( $>95 \%$ purity) were synthesized by GL Biochem. Miglyol 812 was purchased from AXO Industry SA; CellTrace Violet (CTV) from Molecular Probes; albumin from chicken egg white (OVA) from Sigma-Aldrich; EndoGrade Ovalbumin ( $98 \%$ purity, $<1 \mathrm{EU} / \mathrm{mg}$ ) from Hyglos GmbH; mPEG-NHS (MW 5,000, protein dispersibility index $<1.08$, purity $>95 \%$ ) from Nanocs; and FITC-anti-CD3 (clone 17A2), APC/Cy7-anti-CD8 (clone 53.67), PE/Cy7-anti-CD11c (clone N418), APC-anti-CD317 (clone 927), PE-anti-CD45.2 (clone 104), PerCP/ Cy5.5-anti-I-A/I-E (clone M5/114.15.2), PerCP/Cy5.5-anti-CD40 (clone 3/23), PE-anti-CD80 (clone 16-10A1), FITC-anti-CD86 (clone GL-1), APC-anti-CD4 (clone RM4-5), PerCP/Cy5.5-anti-CD45 (clone I3/2.3), PE/Cy7-anti-CD44 (clone IM7), PE-anti-PD 1 (clone 29F.1A12), APC/Cy7-anti-CD4 (clone RM4-5), APC/Cy7-anti-CD11b (clone M1/70), FITC-anti-F4/80 (clone BM8), and APC-anti-CD206 (clone C068C2) from Biolegend. Anti-mouse LAMP1 (clone 1D4B) and anti-mouse EEA1 (clone 14/EEA1) were from BD Biosciences. LIVE/DEAD Fixable Aqua Dead Cell Stain was from Molecular Probes. Cithrol GMO HP was a gift from Croda Europe Ltd. DAMP4 fused with antibody (mAb-DAMP4) was generated as previously described (19, 57). CpG ODN 1668 (catalog tlrl-1668) was purchased from InvivoGen, and administered at $5 \mathrm{nmol}$ per dose. Mice were purchased from the Animal Research Centre or bred at the University of Queensland, QIMR Berghofer, or James Cook University under specific pathogen-free conditions.

Preparation of protein antigen in oil dispersion and TNE. Protein antigen solution $(10 \mathrm{mg} / \mathrm{ml})$ was prepared by dissolving of protein in ultrapure water. Cithrol GMO HP solution (1\%, wt/vol) was prepared by dissolving of Cithrol GMO HP in hexane. Protein and Cithrol GMO HP solutions were mixed in a glass vial using a sonicator for 1 minute at $20 \mathrm{~W}$ to form a stable water-in-oil emulsion. The emulsion was frozen rapidly in dry ice before lyophilization. The antigen-Cithrol GMO HP pellet was dissolved in Miglyol 812 to $5 \mathrm{mg} / \mathrm{ml}$ and used as oil phase. Lyophilized AM1 was dissolved in HEPES containing $\mathrm{ZnCl}_{2}$. Miglyol 812 was added to give an oil volume fraction of 2\% ( $\mathrm{vol} / \mathrm{vol}$ ), then homogenized using an ultrasonicator. TNEs were added to PEGylated DAMP4 solution, then vigorously stirred ( $\mathrm{P}_{20}$-TNE). $\mathrm{mAb}$ DAMP4 was added to $\mathrm{P}_{20}$-TNE and stirred; then $A b-\mathrm{P}_{20}$-TNE was added to PEGylated DAMP4 and stirred. To prepare F-actin-functionalized TNE (F-actin-TNE), actin was polymerized according to a published protocol (40). In brief, rabbit muscle actin was polymerized in F-buffer (10 mM Tris- $\mathrm{HCl}, 50 \mathrm{mM} \mathrm{KCl}, 2 \mathrm{mM} \mathrm{MgCl}_{2}, 1 \mathrm{mM}$ ATP [pH 7.5]). Polymerized F-actin was added to $\mathrm{P}_{20}$-TNE to a final concentration of $50 \mu \mathrm{g} / \mathrm{ml}$ to prepare F-actin-TNE. To prepare WH-TNE, WH peptide was first conjugated to DSPE-PEG-NHS. In brief, WH peptide was mixed with DSPE-PEG-NHS (1.1 to 1 molar ratio) in 25 mM HEPES ( $\mathrm{pH} 8.0$ ) for 24 hours at $4^{\circ} \mathrm{C}$ to allow complete conjugation. AM1 $(400 \mu \mathrm{M})$ was dissolved in $980 \mu \mathrm{l}$ HEPES $(25 \mathrm{mM}, \mathrm{pH}$ 
7.0) containing $\mathrm{ZnCl}_{2}(800 \mu \mathrm{M})$ and DSPE-PEG-WH $(8 \mu \mathrm{M})$. Twenty microliters Miglyol 812 containing peptides/protein was added to yield an oil volume fraction of $2 \%$ (vol/vol). The mixture was homogenized for four 45 -second bursts at $60 \mathrm{~W}$. TNE size was measured by a Zetasizer Nano ZS (Malvern Panalytical). Data analysis with DTS software used the non-negativity constrained least-squares fitting algorithm. Dispersant refractive index and viscosity of the dispersant were assumed to be 1.45 and 1.02 centipoise. Each sample had 10 runs of 10 seconds.

Flow cytometry and confocal microscopy. For TNE in vivo distribution experiments, single-cell suspensions were prepared from spleens and stained for I-A/I-E, CD11c, CD317, CD4, and CD8 surface markers. Dead cells were excluded using LIVE/DEAD Aqua stain. pDCs were gated as I-A/I-E $\mathrm{E}^{+} \mathrm{CD} 11 \mathrm{c}^{\text {int }} \mathrm{CD} 317^{+}, \mathrm{CD} 8^{+} \mathrm{cDCs}$ as I-A/I-E ${ }^{+}$ $\mathrm{CD} 11 \mathrm{c}^{\mathrm{hi}} \mathrm{CD} 8^{+}$, and $\mathrm{CD} 8^{-} \mathrm{cDCs}$ as $\mathrm{I}-\mathrm{A} / \mathrm{I}-\mathrm{E}^{+} \mathrm{CD} 11 \mathrm{c}^{\text {hi }} \mathrm{CD} 8^{-}$. For FACS analysis of tumors, orthotopic PyMT-mChOVA and 4T1.2 mammary tumors were excised and single-cell suspensions were made using collagenase D ( $2 \mathrm{mg} / \mathrm{ml}$; Roche), dispase ( $2 \mathrm{mg} / \mathrm{ml}$; Gibco), and DNase I $(0.1 \mathrm{mg} / \mathrm{ml}$; Roche) digestion in RPMI supplemented with $10 \%$ FCS. After 40 seconds at $37^{\circ} \mathrm{C}$ in a shaking bath, the cell suspension was $40-\mu \mathrm{m}$-filtered and washed in cold PBS. All cell suspensions were first incubated on ice with a 1:1,000 dilution of LIVE/DEAD Aqua for 15 minutes and then incubated with rat anti-mouse Fc $\gamma \mathrm{III} / \mathrm{II}$ receptor (CD16/CD32) blocking antibodies $(4 \mu \mathrm{g} / \mathrm{ml}$; BD Biosciences) for 15 minutes on ice. Thereafter cells were stained for CD 45, CD11b, F4/80, CD11c, CD206, CD4, and CD8 surface markers at $4^{\circ} \mathrm{C}$ for 40 minutes, then washed with PBS containing 2 mM EDTA and 0.5\% BSA. For intracellular staining, samples were fixed, permeabilized, and stained using the FoxP3 transcription factor staining buffer set (eBioscience). Samples were acquired on a Beckman Coulter CytoFLEX.

For confocal microscopy, splenic CD11 $\mathrm{c}^{+}$DCs purified from C57BL/6 mice were plated on coverslips and incubated with DiIlabeled Clec $9 \mathrm{~A}-\mathrm{TNE}$ or isotype-TNE for 1 hour at $37^{\circ} \mathrm{C}$. After washing to remove unbound TNE, cells were incubated in media overnight. Cells were fixed with $4 \%$ paraformaldehyde and permeabilized with $100 \mu \mathrm{g} / \mathrm{ml}$ digitonin (Invitrogen) and stained with rat anti-mouse LAMP1 or rat anti-mouse EEA1, then Alexa Fluor 488-secondary antibody and DAPI (Molecular Probes). Mounted glass coverslips were imaged on an Apotome microscope (Carl Zeiss).

In vitro DC stimulation with TNE. Splenic CD11 $\mathrm{c}^{+} \mathrm{DCs}$ were purified from C57BL/6, CD $40^{-/-}$, or TLR9 $9^{-/-}$mice $(58,59)$. CD $8^{+}$and $\mathrm{CD} 4^{+}$ T cells purified from lymph nodes and spleens of OT-I and OT-II transgenic mice by negative selection were labeled with CTV. CTV-labeled T cells $\left(2 \times 10^{4}\right)$ were cultured with $10^{5} \mathrm{DCs}$, with or without $2 \mu \mathrm{l} \mathrm{TNE}$, for 16 hours. Cells were washed intensively before staining and flow cytometry analysis. Secretion of IFN- $\alpha$ in supernatants was assessed by ELISA (VeriKine Mouse IFN Alpha ELISA Kit, PBL Assay Science).

In vivo proliferation assays of transgenic $T$ cells. B6.SJL-Ptprc ${ }^{a}$ mice were injected i.v. with $3 \times 10^{6} \mathrm{CTV}$-labeled lymph node cells harvested from OT-I or OT-II transgenic mice. One day later, these recipient mice were injected i.v. with $200 \mu$ l OVA-Clec9A-TNE, OVA-isotypeTNE, Clec9A-TNE, or $5 \mu \mathrm{g}$ soluble OVA protein. Six days later, proliferation of $\mathrm{CD}^{+} \mathrm{CD} 45.2^{+} \mathrm{CD}^{+} \mathrm{OT}-\mathrm{I}$ or $\mathrm{CD}^{+} \mathrm{CD} 45.2^{+} \mathrm{CD} 4^{+} \mathrm{OT}-\mathrm{II} \mathrm{T}$ cells was determined by dilution of CTV fluorescence.

In vivo CTL assay. Recipient C57BL/6, Casp1 ${ }^{--}$, IFNAR1 ${ }^{-/}$, or MyD88/Trif ${ }^{--}$mice were injected i.v. with $200 \mu$ l OVA-Clec9A-TNE or OVA-isotype-TNE (both formulated with $5 \mu$ g of OVA), Clec9A-TNE, or soluble OVA (100 $\mu \mathrm{g})$ (60). After 5 days, CTL targets were prepared. Single-cell suspensions of splenocytes from C57BL/6 mice were divided into 2 parts. One was pulsed with $1 \mu \mathrm{g} / \mathrm{ml} \mathrm{OVA}_{257-264}$ and labeled with $5 \mu \mathrm{M}$ of CTV (CTV ${ }^{\text {hi }}$ population). The other was labeled with 0.5 $\mu \mathrm{M}$ of CTV (CTV ${ }^{\mathrm{lo}}$ population). Equal numbers of cells from each were pooled, and $10^{7}$ cells were injected i.v. into recipient mice. Twenty hours after injection, the relative proportion of $\mathrm{CTV}^{\text {hi }}$ to $\mathrm{CTV}^{\text {lo }}$ cells was determined in lymph nodes and spleens by flow cytometry. Percentage (\%) specific lysis in vivo was calculated by [1 - ( $r$ unprimed/ $r$ primed) $] \times 100$, where $r=\% \mathrm{CTV}^{\mathrm{lo}} / \% \mathrm{CTV}^{\text {hi }}$ for each mouse. For CTL assay using TLR4 ${ }^{--}$mice, target cells were labeled with the same concentrations of CFSE.

Immunization using OVA-Clec9A-TNE and Clec9A-OVA. Constructs of OVA conjugated to anti-Clec9A mAb (Clec9A-OVA) or isotype $\mathrm{mAb}$ (isotype-OVA) were prepared as previously described (19, 57). C57BL/6 mice were injected i.v. with $5 \mu \mathrm{g}$ Clec9A-OVA, OVAClec9A-TNE, isotype-OVA, OVA-isotype-TNE, or PBS in the absence of adjuvant. Serum anti-OVA Ig reactivity was measured 1, 2, and 3 weeks later by ELISA as previously described (19).

ELISA. IFN- $\alpha$ levels were determined in mouse serum or primary cell culture media using the VeriKine Mouse IFN Alpha ELISA Kit.

Mouse models. For therapeutic experiments in breast cancer models, C57BL/6 mice were inoculated orthotopically with $10^{6}$ AT3-OVA or $1.5 \times 10^{6}$ PyMT-mChOVA cells into the mammary gland. For therapeutic experiments in the melanoma model, C57BL/6 mice were inoculated s.c. with $2.5 \times 10^{4}$ B16-F10 melanoma cells into the flank and randomly distributed into treatment groups. For therapeutic experiments in the HPV-related cancer model, $10^{5} \mathrm{TC} 1$ cells were inoculated s.c. into the flank of $\mathrm{C} 57 \mathrm{BL} / 6$ mice. Tumor volumes were measured with calipers and calculated using the formula $\left(A \times B^{2}\right) / 2(A$ as the largest and $B$ as the smallest diameter of the tumor). In therapeutic experiments in the PyMT-mChOVA model, mice received 1 dose of OVAClec9A-TNE or OVA-isotype-TNE ( 5 g of OVA per mouse i.v.) either 8 or 14 days after tumor inoculation. In therapeutic experiments in the B16-F10 model, mice received 4 weekly i.v. doses of G-actin-TNE or WH-actin-TNE encapsulating E6/E7 protein or a pool of 6 neoepitopes, respectively (10 $\mu \mathrm{g}$ of mutated epitopes per mouse), commencing 2 days after tumor inoculation. Peptides used in B16-F10 studies included FRRKAFLHWYTGEAMDEMEFTEAESNM (MUT20), STANYNTSHLNNDVWQIFENPVDWKEK (MUT25), PSKPSFQEFVDWENVSPELNSTDQPFL (MUT30), DSGSPFPAAVILRDALHMARGLKYLHQ (MUT33), CGTAFFINFIAIYHHASRAIPFGTMVA (MUT36), EFKHIKAFDRTFANNPGPMVVFATPGM (MUT44), and ATFKNWPFL (survivin ${ }_{20-28}$ universal peptide).

Expression and purification of E6/E7 fusion protein. E6/E7 fusion protein was prepared as previously described (61). In brief, E. coli BL21(DE3) cells transformed with vector encoding E6/E7 fusion protein were used to express the recombinant protein. LB plates containing kanamycin sulfate were streaked, and a single colony was selected and inoculated into LB media containing kanamycin, and expanded. Protein expression was induced with isopropyl- $\beta$-thiogalactopyranoside (IPTG). Cell pellets were stored at $-80^{\circ} \mathrm{C}$ until further use. Proteins were purified by Ni-NTA agarose (Qiagen Ni-NTA Fast Start Kit). Endotoxin was eliminated using Pierce High Capacity Endotoxin Removal Spin Columns. Protein concentration was measured by a Thermo Fisher Scientific NanoDrop ND-1000 spectrophotometer before encapsulation into TNE. 
Splenocyte stimulation and CD107/IFN- $\gamma / I L-10$ analysis. Spleens were removed from tumor-bearing mice and mechanically disrupted through a 70- $\mu \mathrm{m}$ cell strainer into a single-cell suspension. Erythrocytes were lysed by ACK lysis buffer. Splenocytes $\left(10^{6}\right.$ cells per $200 \mu \mathrm{l}$ per well) were seeded into a 96-well culture plate and cultured with or without individual epitopes $(10 \mu \mathrm{g} / \mathrm{ml})$ at $37^{\circ} \mathrm{C}$ for 8 hours. BV421anti-mouse CD107a (1D4B, Biolegend) and BV421-anti-CD107b (ABL-93, BD Biosciences) were added to evaluate degranulation of cytotoxic granules. T cells producing IL-10 and IFN- $\gamma$ were detected by intracellular cytokine staining following addition of GolgiPlug (catalog 555029, BD Biosciences) during the last 5 hours of stimulation. Staining was performed using FoxP3/Transcription Factor Staining Buffer Set (catalog 00-5523-00, eBioscience).

ELISPOT. The IFN- $\gamma$ release enzyme-linked ImmunoSpot (ELISPOT) (41) was carried out using monocytes isolated by a 2-hour plastic adherence step from naive syngeneic splenocytes as antigen-presenting cells and $\mathrm{CD}^{+}$peripheral blood $\mathrm{T}$ lymphocytes from tumor-bearing mice using the Pan T Cell Isolation Kit II (Miltenyi Biotec) as responders. Effector and responder cells $\left(25,000\right.$ each) were cultured at $37^{\circ} \mathrm{C}$ for 48 hours in the presence of anti-IFN- $\gamma$-coated $(10 \mu \mathrm{g} / \mathrm{ml}$, clone AN18) Multiscreen 96-well plates (Millipore). Cytokine secretion was detected with an anti-IFN- $\gamma$ antibody $(1 \mu \mathrm{g} / \mathrm{ml}$, clone R4-6A2) stimulated without or with $10 \mu \mathrm{g} / \mathrm{ml}$ of peptide. All samples were tested in triplicate.

Statistics. Results are presented as mean with SD of each group. Data were statistically analyzed by ordinary 1-way or, where appropriate, 2-way ANOVA and Tukey's multiple-comparisons test, using GraphPad Prism software. $P$ values of 0.05 were considered significant. Survival analyses used the Mantel-Cox log-rank test.

Study approval. The animal experiments were approved by Animal Ethics Committees of the University of Queensland, Brisbane, Australia, and James Cook University, Townsville, Australia.

\section{Author contributions}

BZ, APJM, RM, IC, ML, RD, and RT designed the study. BZ, MT, $\mathrm{DM}$, and KMT conducted and analyzed experiments. AG, KM, AGB, IC, and ML provided reagents. BZ, IC, RM, ML, RD, and RT wrote the manuscript. All authors read and approved the final draft of the manuscript.

\section{Acknowledgments}

The work was supported by National Health and Medical Research Council (NHMRC) grants 1083192, 1082665, and 1083747 , and by a grant from the Australian Skin and Skin Cancer Centre. RT was supported by Arthritis Queensland and an NHMRC Research Fellowship. ML and IC were supported by a Bill and Janina Amiet Fellowship. We thank Christian Engwerda (QIMR Berghofer) and Ian Frazer (Diamantina Institute, University of Queensland) for supplying mice and Hendrik Nel for technical assistance.

Address correspondence to: Riccardo Dolcetti or Ranjeny Thomas, The University of Queensland Diamantina Institute, Level 7, 37 Kent St., Translational Research Institute, Woolloongabba, QLD 4102 Australia. Phone: 61.7.34436953; Email: r.dolcetti@ uq.edu.au (R. Dolcetti). Phone: 61.7.34436960; Email: Ranjeny. thomas@uq.edu.au (R.Thomas).

APJM's present address is: Faculty of Engineering, Computer and Mathematical Sciences, The University of Adelaide, Adelaide, Australia.

AG's present address is: Mater Research Institute, Translational Research Institute, Brisbane, Queensland, Australia.
1. van der Burg SH, Arens R, Ossendorp F, van Hall T, Melief CJ. Vaccines for established cancer: overcoming the challenges posed by immune evasion. Nat Rev Cancer. 2016;16(4):219-233.

2. van Elsas A, et al. Elucidating the autoimmune and antitumor effector mechanisms of a treatment based on cytotoxic T lymphocyte antigen-4 blockade in combination with a B16 melanoma vaccine: comparison of prophylaxis and therapy. JExp Med. 2001;194(4):481-489.

3. Duraiswamy J, Kaluza KM, Freeman GJ, Coukos G. Dual blockade of PD-1 and CTLA-4 combined with tumor vaccine effectively restores T-cell rejection function in tumors. Cancer Res. 2013;73(12):3591-3603.

4. van den Eertwegh AJ, et al. Combined immunotherapy with granulocyte-macrophage colony-stimulating factor-transduced allogeneic prostate cancer cells and ipilimumab in patients with metastatic castration-resistant prostate cancer: a phase 1 dose-escalation trial. Lancet Oncol. 2012;13(5):509-517.

5. Le DT, Jaffee EM. Next-generation cancer vaccine approaches: integrating lessons learned from current successes with promising biotechnologic advances. J Natl Compr Canc Netw. 2013;11(7):766-772.

6. Kreiter S, et al. Mutant MHC class II epitopes drive therapeutic immune responses to cancer.
Nature. 2015;520(7549):692-696.

7. Kranz LM, et al. Systemic RNA delivery to dendritic cells exploits antiviral defence for cancer immunotherapy. Nature. 2016;534(7607):396-401.

8. Kenter GG, et al. Phase I immunotherapeutic trial with long peptides spanning the E6 and E7 sequences of high-risk human papillomavirus 16 in end-stage cervical cancer patients shows low toxicity and robust immunogenicity. Clin Cancer Res. 2008;14(1):169-177.

9. Kenter GG, et al. Vaccination against HPV-16 oncoproteins for vulvar intraepithelial neoplasia. NEngl JMed. 2009;361(19):1838-1847.

10. van Poelgeest MI, et al. HPV16 synthetic long peptide (HPV16-SLP) vaccination therapy of patients with advanced or recurrent HPV16-induced gynecological carcinoma, a phase II trial. J Transl Med. 2013;11:88.

11. Hildner K, et al. Batf3 deficiency reveals a critical role for CD8alpha+ dendritic cells in cytotoxic T cell immunity. Science. 2008;322(5904):1097-1100.

12. Jongbloed SL, et al. Human CD141+ (BDCA-3)+ dendritic cells (DCs) represent a unique myeloid DC subset that cross-presents necrotic cell antigens. JExp Med. 2010;207(6):1247-1260.

13. Segura E, Amigorena S. Cross-presentation in mouse and human dendritic cells. Adv Immunol. 2015;127:1-31.
14. Fuertes MB, et al. Host type I IFN signals are required for antitumor $\mathrm{CD}^{+} \mathrm{T}$ cell responses through CD $8 \alpha^{+}$dendritic cells. JExp Med. 2011;208(10):2005-2016.

15. Broz ML, et al. Dissecting the tumor myeloid compartment reveals rare activating antigenpresenting cells critical for $\mathrm{T}$ cell immunity. Cancer Cell. 2014;26(5):638-652.

16. Sánchez-Paulete AR, et al. Cancer immunotherapy with immunomodulatory anti-CD137 and anti-PD-1 monoclonal antibodies requires BATF3-dependent dendritic cells. Cancer Discov. 2016;6(1):71-79.

17. Bennett SR, Carbone FR, Karamalis F, Flavell RA, Miller JF, Heath WR. Help for cytotoxic-T-cell responses is mediated by CD40 signalling. Nature. 1998;393(6684):478-480.

18. Bennett SR, Carbone FR, Karamalis F, Miller JF, Heath WR. Induction of a CD8 ${ }^{+}$cytotoxic T lymphocyte response by cross-priming requires cognate CD4 ${ }^{+}$T cell help. JExp Med.1997;186(1):65-70.

19. Caminschi I, et al. The dendritic cell subtyperestricted C-type lectin Clec9A is a target for vaccine enhancement. Blood. 2008;112(8):3264-3273.

20. Idoyaga J, Suda N, Suda K, Park CG, Steinman RM. Antibody to Langerin/CD207 localizes large numbers of $\mathrm{CD} 8 \alpha^{+}$dendritic cells to the marginal zone of mouse spleen. Proc Natl Acad Sci U S A. 2009;106(5):1524-1529. 
21. Jiang W, Kim BY, Rutka JT, Chan WC. Nanoparticle-mediated cellular response is size-dependent. Nat Nanotechnol. 2008;3(3):145-150.

22. Lahoud $\mathrm{MH}$, et al. Targeting antigen to mouse dendritic cells via Clec9A induces potent CD4 T cell responses biased toward a follicular helper phenotype. J Immunol. 2011;187(2):842-850.

23. Lahoud $\mathrm{MH}$, et al. The C-type lectin Clec12A present on mouse and human dendritic cells can serve as a target for antigen delivery and enhancement of antibody responses. JImmunol. 2009;182(12):7587-7594.

24. Sancho D, et al. Tumor therapy in mice via antigen targeting to a novel, DC-restricted C-type lectin. JClin Invest. 2008;118(6):2098-2110.

25. Yan Z, et al. A novel peptide targeting Clec9a on dendritic cell for cancer immunotherapy. Oncotarget. 2016;7(26):40437-40450.

26. O'Hagan DT, Tsai T, Reed S. Emulsion-based adjuvants for improved influenza vaccines. In: Rappuoli R, Del Giudice G, eds. Influenza Vaccines for the Future. Basel, Switzerland: Springer Basel; 2011:327-357.

27. Calabro S, et al. Vaccine adjuvants alum and MF59 induce rapid recruitment of neutrophils and monocytes that participate in antigen transport to draining lymph nodes. Vaccine. 2011;29(9):1812-1823.

28. Seubert A, Monaci E, Pizza M, O’Hagan DT, Wack $A$. The adjuvants aluminum hydroxide and MF59 induce monocyte and granulocyte chemoattractants and enhance monocyte differentiation toward dendritic cells. J Immunol. 2008;180(8):5402-5412.

29. Stanberry LR, et al. Safety and immunogenicity of a novel nanoemulsion mucosal adjuvant W805EC combined with approved seasonal influenza antigens. Vaccine. 2012;30(2):307-316.

30. Boyce TG, et al. Safety and immunogenicity of adjuvanted and unadjuvanted subunit influenza vaccines administered intranasally to healthy adults. Vaccine. 2000;19(2-3):217-226.

31. Zeng BJ, et al. Receptor-specific delivery of protein antigen to dendritic cells by a nanoemulsion formed using top-down non-covalent click self-assembly. Small. 2013;9(22):3736-3742.

32. Burgdorf S, Kautz A, Böhnert V, Knolle PA, Kurts C. Distinct pathways of antigen uptake and intracellular routing in CD4 and CD8 T cell activation. Science. 2007;316(5824):612-616.

33. Chatterjee $\mathrm{B}$, et al. Internalization and endosomal degradation of receptor-bound antigens regulate the efficiency of cross presentation by human dendritic cells. Blood. 2012;120(10):2011-2020.
34. Nair-Gupta P, et al. TLR signals induce phagosomal MHC-I delivery from the endosomal recycling compartment to allow cross-presentation. Cell. 2014;158(3):506-521.

35. Cella M, et al. Plasmacytoid monocytes migrate to inflamed lymph nodes and produce large amounts of type I interferon. Nat Med. 1999;5(8):919-923.

36. Schoenberger SP, Toes RE, van der Voort EI, Offringa R, Melief CJ. T-cell help for cytotoxic $\mathrm{T}$ lymphocytes is mediated by CD40-CD4OL interactions. Nature. 1998;393(6684):480-483.

37. Guiducci C, et al. Properties regulating the nature of the plasmacytoid dendritic cell response to Toll-like receptor 9 activation. J Exp Med. 2006;203(8):1999-2008.

38. Honda K, et al. Spatiotemporal regulation of MyD88-IRF-7 signalling for robust type-I interferon induction. Nature. 2005;434(7036):1035-1040.

39. Engelhardt JJ, et al. Marginating dendritic cells of the tumor microenvironment cross-present tumor antigens and stably engage tumor-specific T cells. Cancer Cell. 2012;21(3):402-417.

40. Ahrens S, et al. F-actin is an evolutionarily conserved damage-associated molecular pattern recognized by DNGR-1, a receptor for dead cells. Immunity. 2012;36(4):635-645.

41. Kreiter S, et al. Mutant MHC class II epitopes drive therapeutic immune responses to cancer. Nature. 2015;520(7549):692-696.

42. Lladser A, et al. Intradermal DNA electroporation induces survivin-specific CTLs, suppresses angiogenesis and confers protection against mouse melanoma. Cancer Immunol Immunother. 2010;59(1):81-92.

43. Zelenay S, et al. The dendritic cell receptor DNGR-1 controls endocytic handling of necrotic cell antigens to favor cross-priming of CTLs in virus-infected mice. J Clin Invest. 2012;122(5):1615-1627.

44. Kokate RA, et al. Rationalizing the use of functionalized poly-lactic-co-glycolic acid nanoparticles for dendritic cell-based targeted anticancer therapy. Nanomedicine (Lond). 2016;11(5):479-494.

45. Luo Z, et al. Nanovaccine loaded with poly I:C and STAT3 siRNA robustly elicits anti-tumor immune responses through modulating tumor-associated dendritic cells in vivo. Biomaterials. 2015;38:50-60.

46. Pardee AD, et al. Route of antigen delivery impacts the immunostimulatory activity of dendritic cell-based vaccines for hepatocellular carcinoma. J Immunother Cancer. 2015;3:32.

47. Sistigu A, et al. Cancer cell-autonomous contribu- tion of type I interferon signaling to the efficacy of chemotherapy. Nat Med.2014;20(11):1301-1309.

48. Nierkens S, et al. Immune adjuvant efficacy of $\mathrm{CpG}$ oligonucleotide in cancer treatment is founded specifically upon TLR9 function in plasmacytoid dendritic cells. Cancer Res. 2011;71(20):6428-6437.

49. Cella M, Facchetti F, Lanzavecchia A, Colonna M. Plasmacytoid dendritic cells activated by influenza virus and CD4OL drive a potent TH1 polarization. Nat Immunol. 2000;1(4):305-310.

50. Welters MJ, et al. Vaccination during myeloid cell depletion by cancer chemotherapy fosters robust T cell responses. Sci Transl Med. 2016;8(334):334ra52.

51. Maldonado L, et al. Intramuscular therapeutic vaccination targeting HPV16 induces T cell responses that localize in mucosal lesions. Sci Transl Med. 2014;6(221):221ra13.

52. Welters MJ, et al. Success or failure of vaccination for HPV16-positive vulvar lesions correlates with kinetics and phenotype of induced T-cell responses. Proc Natl Acad Sci U S A. 2010;107(26):11895-11899.

53. Sahin U, et al. Personalized RNA mutanome vaccines mobilize poly-specific therapeutic immunity against cancer. Nature. 2017;547(7662):222-226.

54. Ott PA, et al. An immunogenic personal neoantigen vaccine for patients with melanoma. Nature. 2017;547(7662):217-221.

55. Backert L, Kohlbacher O. Immunoinformatics and epitope prediction in the age of genomic medicine. Genome Med. 2015;7:119.

56. Dexter AF, Malcolm AS, Middelberg AP. Reversible active switching of the mechanical properties of a peptide film at a fluid-fluid interface. Nat Mater. 2006;5(6):502-506.

57. Caminschi I, et al. Antibody responses initiated by Clec9A-bearing dendritic cells in normal and Batf3(-/-) mice. Mol Immunol. 2012;50(1-2):9-17.

58. Hemmi H, et al. A Toll-like receptor recognizes bacterial DNA. Nature. 2000;408(6813):740-745.

59. Yamamoto M, et al. Role of adaptor TRIF in the MyD88-independent toll-like receptor signaling pathway. Science. 2003;301(5633):640-643.

60. Kayagaki N, et al. Non-canonical inflammasome activation targets caspase-11. Nature. 2011;479(7371):117-121.

61. Edwards SJ, Margetts MB, Hocking DM, Moloney $\mathrm{MBH}$, Rothel LJ, Webb EA. Design of a candidate recombinant therapeutic vaccine for cervical cancer. In: Pandalai SG, ed. Recent Research Developments in Biotechnology and Bioengineering. Kerala, India: Research Singpost; 1998; 343-356. 